\title{
Total Synthesis and Elucidation of Absolute Configuration of the Diterpene Tonantzitlolone
}

Christian Jasper, Rüdiger Wittenberg, Monika Quitschalle, Jasmin Jakupovic, Andreas Kirschning* 


\section{Supporting Information}

General Methods. ${ }^{1} \mathrm{H}$ NMR, ${ }^{13} \mathrm{C}$ NMR and ${ }^{1} \mathrm{H},{ }^{13} \mathrm{C}-\mathrm{COSY}$ as well as NOESY spectra were measured on Avance 200/DPX (Bruker) with $200 \mathrm{MHz}(50 \mathrm{MHz})$, Avance 400/DPX (Bruker) $400 \mathrm{MHz}(100 \mathrm{MHz})$ and Avance 500/DRX (Bruker) respectively, using tetramethylsilane as the internal standard. If not otherwise noted, $\mathrm{CDCl}_{3}$ is the solvent for all NMR experiments. Multiplicities are described using the following abbreviations: $\mathrm{s}=$ singlet, $\mathrm{d}$ $=$ doublet, $\mathrm{t}=$ triplet, $\mathrm{q}=$ quartet, $\mathrm{m}=$ multiplet, $\mathrm{br}=$ broad. Chemical shift values of ${ }^{13} \mathrm{C}$ NMR spectra are reported as values in $\mathrm{ppm}$ relative to residual $\mathrm{CHCl}_{3}(77 \mathrm{ppm})$ or $\mathrm{CD}_{3} \mathrm{OD}$ (49) as internal standards. The multiplicities refer to the resonances in the off-resonance spectra and were elucidated using the distortionless enhancement by polarisation transfer (DEPT) spectral editing technique, with secondary pulses at $90^{\circ}$ and $135^{\circ}$. Mass spectra were recorded on a type LCT-spectrometer (Micromass) and on a type $\mathrm{VG}$ autospec (Micromass). Optical rotations $[\alpha]$ were collected on a Polarimeter 341 (Perkin Elmer) at a wavelength of $589 \mathrm{~nm}$ and are given in $10^{-1} \mathrm{deg} \mathrm{cm}^{2} \mathrm{~g}^{-1}$. All solvents used were of reagent grade and were further dried. Reactions were monitored by thin layer chromatography (tlc) on silica gel $60 \mathrm{~F}^{254}$ (E. Merck, Darmstadt) and spots were detected either by UV-absorption or by charring with $\mathrm{H}_{2} \mathrm{SO}_{4} / 4$-methoxybenzaldehyde in methanol. Preparative column chromatography was performed on silica gel 60 (E. Merck, Darmstadt).

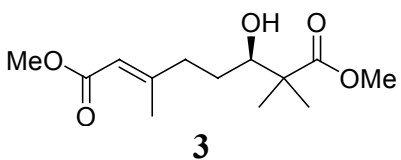

$(E, 6 R)$-Hydroxy-3,7,7-trimethyl-oct-2-enedioic acid dimethylester 3. A flame-dried 3-neck $500 \mathrm{~mL}$ round bottom flask equipped with a pressure equalized dropping funnel was charged with $N$-tosylated $L$-valin $(7.85 \mathrm{~g}, 28.9$ mmol) and $290 \mathrm{~mL}$ of anhydrous $\mathrm{CH}_{2} \mathrm{Cl}_{2}$. A borane-THF complex solution (28.9 mL, $1 \mathrm{M}$ THF) was added dropwise and the solution was allowed to stir for additional 20 minutes. Then the reaction mixture was cooled to $-78^{\circ} \mathrm{C}$, and aldehyde 5 (4.52 $\mathrm{g}, 28.9 \mathrm{mmol})$ in $30 \mathrm{~mL}$ of dry $\mathrm{CH}_{2} \mathrm{Cl}_{2}$ was introduced, followed by ketene acetal 6 (7.56 g, $43.4 \mathrm{mmol}$ ) in $30 \mathrm{~mL}$ of dry $\mathrm{CH}_{2} \mathrm{Cl}_{2}$. After complete addition the solution was warmed slowly to $-30^{\circ} \mathrm{C}$ and cautiously terminated with $50 \mathrm{~mL}$ of phosphate buffer solution $(\mathrm{pH}=7)$. The mixture was warmed to room temperature and the layers were separated. The aqueous layer was extracted with $\mathrm{CH}_{2} \mathrm{Cl}_{2}(3 \times 50 \mathrm{~mL})$, and the combined organic solutions were dried over $\mathrm{MgSO}_{4}$ and concentrated under reduced pressure. The residue was treated with hexanes $(100 \mathrm{~mL})$ and the solution was filtered to remove the amino acid. The filtrate was concentrated under reduced pressure and taken up in $\mathrm{MeOH}(50 \mathrm{~mL})$, and a catalytic amount of acetyl chloride was added to cleave the silyl ether. After 15 minutes the solution was concentrated under reduced pressure again and purified by flash chromatography (hexanes/EtOAc, 4:1) to furnish the desired aldol product, $5.45 \mathrm{~g}(21.1 \mathrm{mmol}, 73 \%)$, as an oil.
Data are: TLC $R_{f}=0.17$ (hexanes/EtOAc, 4:1); $[\alpha]_{\mathrm{D}}{ }^{20}=$ $+20.3^{\circ}\left(\mathrm{c} 1, \mathrm{CHCl}_{3}\right) ;{ }^{1} \mathrm{H} \mathrm{NMR}\left(400 \mathrm{MHz}, \mathrm{CDCl}_{3}\right) \delta 5.67$ $(\mathrm{dt}, J=3.6,1.2 \mathrm{~Hz}, 1 \mathrm{H}), 3.67(\mathrm{~s}, 3 \mathrm{H}), 3.65$ (s, $3 \mathrm{H}), 3.54$ (ddd, $J=10.8,6.9,2.0 \mathrm{~Hz}, 1 \mathrm{H}), 2.69$ (d, $J=6.9 \mathrm{~Hz}, 1 \mathrm{H})$, 2.43 (dddd, $J=14.6,9.7,4.8,1.2 \mathrm{~Hz}, 1 \mathrm{H}$ ), 2.17 (dddd, $J=$ 14.6, 9.6, 6.7, $1.0 \mathrm{~Hz}, 1 \mathrm{H}), 2.13$ (d, $J=1.2 \mathrm{~Hz}, 3 \mathrm{H}), 1.58$ (dddd, $J=13.7,10.8,9.7,6.7, \mathrm{~Hz}, 1 \mathrm{H}$ ), 1.42 (dddd, $J=$ 13.7, 9.6, 4.8, 2.0 Hz, 1 H), 1.16 (s, $3 \mathrm{H}), 1.15(\mathrm{~s}, 3 \mathrm{H}) ;{ }^{13} \mathrm{C}$ NMR $\left(100 \mathrm{MHz}, \mathrm{CDCl}_{3}\right) \delta 178.0,167.1,159.9,115.4$, 75.8, 51.9, 50.7, 47.0, 37.7, 29.3, 22.2, 20.4, 18.8; IR (neat) v 3507, 2979, 2951, 1715, 1648, 1435, 1272, 1223, 1146, 1076, 1023, 860; MS (EI) $m / z(\%) 258\left(\mathrm{M}^{+}, 28\right), 243(25)$, 226 (33), 211 (38), 195 (55), 176 (46), 157 (59), 144 (65), 125 (100), 102 (100), 97 (81), 87 (67), 70 (98); HRMS (EI) found $258.1466 \mathrm{M}^{+}$, calcd 258.1467 for $\mathrm{C}_{13} \mathrm{H}_{22} \mathrm{O}_{5}$.

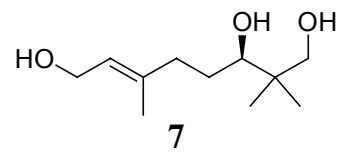

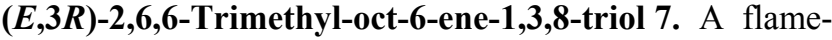
dried $500 \mathrm{~mL}$ round bottom flask equipped with a pressure equalized dropping funnel was charged with $\mathrm{LiALH}_{4}(4 \mathrm{~g}$, $105 \mathrm{mmol}$ ) and $210 \mathrm{~mL}$ of anhydrous $\mathrm{Et}_{2} \mathrm{O}$. The reaction mixture was cooled to $0^{\circ} \mathrm{C}$, and diester 3 (5.45 g, 21.1 $\mathrm{mmol}$ ) in $20 \mathrm{~mL}$ of anhydrous $\mathrm{Et}_{2} \mathrm{O}$ was added dropwise. The mixture was warmed to room temperature in the cold bath and stirred over night. Then $\mathrm{H}_{2} \mathrm{O}(4 \mathrm{~mL})$ was cautiously added, followed by $4 \mathrm{~N}$ aqueous $\mathrm{NaOH}(4 \mathrm{~mL})$ and $\mathrm{H}_{2} \mathrm{O}(8 \mathrm{~mL})$. After $1 \mathrm{~h} \mathrm{Na} \mathrm{Na}_{2} \mathrm{SO}_{4}$ was added and the mixture was filtered. The residue was washed with EtOAc and the combined organic solutions were concentrated under reduced pressure to deliver the triol, $4.27 \mathrm{~g}$ (21.1 mmol, >99\%), as an oil.

Data are: tlc $R_{f}=0.17\left(\right.$ EtOAc); $[\alpha]_{\mathrm{D}}{ }^{20}=+23.6^{\circ}$ (c 1, $\left.\mathrm{CHCl}_{3}\right) ;{ }^{1} \mathrm{H} \mathrm{NMR}\left(400 \mathrm{MHz}, \mathrm{CDCl}_{3}\right) \delta 5.43-5.46(\mathrm{~m}, 1 \mathrm{H})$, 4.16-4.12 (m, $2 \mathrm{H}), 3.57$ (d, $J=10.8 \mathrm{~Hz}, 1 \mathrm{H}), 3.44$ (dd, $J=$ 10.6, 1.7 Hz, $1 \mathrm{H}$ ), 3.39 (d, $J=10.8 \mathrm{~Hz}, 1 \mathrm{H}$ ), 2.27 (ddd, $J$ $=14.0,8.6,5.3 \mathrm{~Hz}, 1 \mathrm{H}), 2.11-2.07(\mathrm{~m}, 1 \mathrm{H}), 1.67$ (bs, 3 H), 1.66-1.61 (m, $1 \mathrm{H}), 1.46$ (dddd, $J=13.8,10.6,8.6,5.3$, $\mathrm{Hz}, 1 \mathrm{H}), 0.89$ (s, $3 \mathrm{H}), 0.85$ (s, $3 \mathrm{H}) ;{ }^{13} \mathrm{C} \mathrm{NMR}(100 \mathrm{MHz}$, $\left.\mathrm{CDCl}_{3}\right) \delta 139.3,123.9,77.9,71.9,59.1,38.5,36.4,29.2$, 22.5, 18.8, 16.1; IR (neat) $v$ 3317, 2954, 2873, 1739, 1442, 1381, 1230, 1034, 1000, 932; MS (EI) $m / z(\%) 202\left(\mathrm{M}^{+}, 1\right)$, 184 (10), 169 (17), 151 (19), 131 (36), 129 (27), 116 (66), 95 (49), 84 (100), 69 (78); HRMS (EI) found 202.1554 $\mathrm{M}^{+}$, calcd 202.1569 for $\mathrm{C}_{11} \mathrm{H}_{22} \mathrm{O}_{3}$.

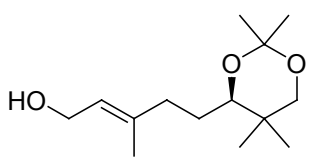

(no compound number was assigned in the manuscript) $(\boldsymbol{E}, \boldsymbol{R})$-Allyl alcohol. A $250 \mathrm{~mL}$ round bottom flask was charged with triol $7(6.57 \mathrm{~g}, 32.5 \mathrm{mmol})$ and $70 \mathrm{~mL}$ of anhydrous DMF. 2,2-Dimethoxy propane $(20 \mathrm{~mL})$ was added at room temperature, followed by a catalytic amount of $p \mathrm{TsOH}$ (62 mg, $0.326 \mathrm{mmol})$. After 30 minutes $\mathrm{H}_{2} \mathrm{O}(20$ 
$\mathrm{mL}$ ) was added to terminate the reaction and to cleave the mixed acetal formed as byproduct. Finally $\mathrm{K}_{2} \mathrm{CO}_{3}$ was added and the layers were separated. The aqueous phase was extracted with MTBE $(5 \times 25 \mathrm{~mL})$, and the combined organic solutions were dried over $\mathrm{MgSO}_{4}$ and concentrated under reduced pressure. The residue was purified by flash column chromatography (hexanes/EtOAc, 3:1) to afford the title compound, $7.46 \mathrm{~g}(30.8 \mathrm{mmol}, 95 \%)$, as an oil.

Data are: tlc $R_{f}=0.41$ (hexanes/EtOAc, 2:1); $[\alpha]_{\mathrm{D}}{ }^{20}=$ $+25.1^{\circ}\left(c 1, \mathrm{CHCl}_{3}\right) ;{ }^{1} \mathrm{H}$ NMR $\left(400 \mathrm{MHz}, \mathrm{CD}_{3} \mathrm{OD}\right) \delta 5.36$ (app dt, $J=6.8,1.3 \mathrm{~Hz}, 1 \mathrm{H}), 4.08(\operatorname{app~d}, J=6.8,2 \mathrm{H})$, $3.64(\mathrm{~d}, J=11.4 \mathrm{~Hz}, 1 \mathrm{H}), 3.54(\mathrm{dd}, J=10.3,1.7 \mathrm{~Hz}, 1 \mathrm{H})$, $3.23(\mathrm{~d}, J=11.4 \mathrm{~Hz}, 1 \mathrm{H}), 2.19-2.15$ (m, $1 \mathrm{H}), 2.01-1.97$ (m, $1 \mathrm{H}), 1.65$ (s, $3 \mathrm{H}), 1.58$ (dddd, $J=13.7,9.1,7.5,1.7$ $\mathrm{Hz}, 1 \mathrm{H}), 1.40$ (s, $3 \mathrm{H}), 1.38-1.34(\mathrm{~m}, 1 \mathrm{H}), 1.34$ (s, $3 \mathrm{H})$, $0.98(\mathrm{~s}, 3 \mathrm{H}), 0.73(\mathrm{~s}, 3 \mathrm{H}) ;{ }^{13} \mathrm{C}$ NMR $\left(100 \mathrm{MHz}, \mathrm{CD}_{3} \mathrm{OD}\right)$ $\delta 139.2,125.4,100.0,77.5,73.0,59.4,36.9,33.7,30.0$, 28.2, 21.9, 19.4, 18.5, 16.1; IR (neat) v 3420, 2990, 2951, 2856, 1463, 1391, 1378, 1360, 1263, 1228, 1198, 1171, $1157,1102,1072,1050,1004,937,921,904,848$.

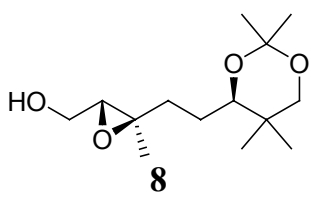

Epoxide 8. A flame-dried 3-neck $250 \mathrm{~mL}$ round bottom flask equipped with a pressure equalized dropping funnel was charged with powdered $4 \AA$ molecular sieves $(816 \mathrm{mg}$ ) and $40 \mathrm{~mL}$ of dry $\mathrm{CH}_{2} \mathrm{Cl}_{2}$. The solution was cooled to -15 to $-20^{\circ} \mathrm{C}$, and then $\mathrm{Ti}(i \mathrm{PrO})_{4}(296 \mu \mathrm{L}, 1 \mathrm{mmol})$ was added, followed by (-)-diethyl- $D$-tartrate $(205 \mu \mathrm{L}, 1.2 \mathrm{mmol})$ in 7 $\mathrm{mL}$ of dry $\mathrm{CH}_{2} \mathrm{Cl}_{2}$ and tbutyl hydroperoxide $(3.64 \mathrm{~mL}, 5.5$ $\mathrm{M}$ in $\mathrm{CH}_{2} \mathrm{Cl}_{2}$ ). After 30 minutes the suspension was cooled to $-25^{\circ} \mathrm{C}$ before the above synthesized allylic alcohol $(2.42$ g, $10 \mathrm{mmol}$ ) dissolved in $50 \mathrm{~mL}$ of dry $\mathrm{CH}_{2} \mathrm{Cl}_{2}$ was introduced dropwise via a dropping funnel. The reaction was allowed to stir for $16 \mathrm{~h}$ at $-25^{\circ} \mathrm{C}$ and then filtered through a plug of celite. The filtrate was mixed with $20 \mathrm{~mL}$ of $\mathrm{H}_{2} \mathrm{O}$ and warmed to room temperature, then $30 \mathrm{~mL}$ of brine-saturated aqueous $\mathrm{NaOH}(30 \%)$ was added and the mixture was vigorously stirred for $1 \mathrm{~h}$. The aqueous layer was separated and extracted with $\mathrm{CH}_{2} \mathrm{Cl}_{2}(5 \times 20 \mathrm{~mL})$. The combined organic layers were dried over anhydrous $\mathrm{MgSO}_{4}$ and concentrated under reduced pressure. Flash chromatography (hexanes/EtOAc, 3:1) afforded the title compound as an oil along with a small amount of recovered starting material. The procedure was repeated twice with allylic alcohol (2.42 g, $\left.2.2 \mathrm{~g}, \sum=7.04 \mathrm{~g}, 29 \mathrm{mmol}\right)$ as described above. A final epoxidation was carried out with the combined recovered starting materials (1.22 g) to deliver an overall yield of $5.97 \mathrm{~g}(23.1 \mathrm{mmol}, 80 \%)$.

Data are: tlc $R_{f}=0.27$ (hexanes/EtOAc, $2 / 1$ ); $[\alpha]_{\mathrm{D}}{ }^{20}=$ $+22.9^{\circ}\left(c 1, \mathrm{CHCl}_{3}\right) ;{ }^{1} \mathrm{H}$ NMR $\left(400 \mathrm{MHz}, \mathrm{CD}_{3} \mathrm{OD}\right) \delta 3.70$ $(\mathrm{dd}, J=12.2,5.0 \mathrm{~Hz}, 1 \mathrm{H}), 3.65$ (d, $J=11.4 \mathrm{~Hz}, 1 \mathrm{H}), 3.61$ $(\mathrm{dd}, J=12.2,6.3 \mathrm{~Hz}, 1 \mathrm{H}), 3.56(\mathrm{dd}, J=9.9,1.8 \mathrm{~Hz}, 1 \mathrm{H})$, $3.23(\mathrm{~d}, J=11.4 \mathrm{~Hz}, 1 \mathrm{H}), 2.91(\mathrm{dd}, J=6.3,5.0 \mathrm{~Hz}, 1 \mathrm{H})$, 1.89-1.80 (m, $1 \mathrm{H}), 1.59-1.50(\mathrm{~m}, 1 \mathrm{H}), 1.42$ (bs, $3 \mathrm{H})$, 1.41-1.35 (m, $2 \mathrm{H}), 1.33$ (bs, $3 \mathrm{H}), 1.26$ (s, $3 \mathrm{H}), 0.99$ (s, 3 $\mathrm{H}), 0.75(\mathrm{~s}, 3 \mathrm{H}) ;{ }^{13} \mathrm{C}$ NMR (100 MHz, CD $\mathrm{CD}_{3} \mathrm{O} 100.0$,
78.0, 72.9, 64.2, 61.8, 61.7, 35.9, 33.8, 30.0, 25.7, 21.9, 19.4, 18.4, 17.0; IR (neat) v 3427, 2990, 2958, 2862, 1462, 1392, 1378, 1360, 1262, 1198, 1157, 1105, 1078, 1041, $1009,920,899,855$.

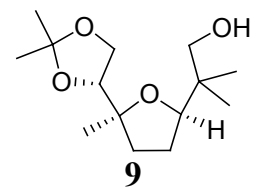

South fragment 9. A $250 \mathrm{~mL}$ round bottom flask was charged with epoxide 8 (2.72 g, $10.5 \mathrm{mmol})$ and $100 \mathrm{~mL}$ of dry $\mathrm{CH}_{2} \mathrm{Cl}_{2}$. A solution of ethylene glycol $(704 \mu \mathrm{L}, 12.6$ mmol) in $55 \mathrm{~mL}$ of dry $\mathrm{CH}_{2} \mathrm{Cl}_{2} / \mathrm{THF}$ (10:1) was added, followed by dry $p \mathrm{TsOH}(181 \mathrm{mg}, 1.05 \mathrm{mmol})$. After 30 minutes, complete conversion of the starting material was observed before 2,2-dimethoxy propane $(17 \mathrm{~mL})$ was introduced. The solution was allowed to stir for $16 \mathrm{~h}$ and then terminated by addition of saturated $\mathrm{NaHCO}_{3}$ solution $(30 \mathrm{~mL})$. The aqueous layer was separated and extracted with $\operatorname{MTBE}(5 \times 20 \mathrm{~mL})$. The combined organic layers were dried over anhydrous $\mathrm{MgSO}_{4}$ and concentrated under reduced pressure. Flash chromatography (hexanes/EtOAc,

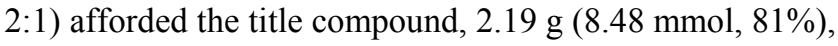
as an oil.

Data are: tlc $R_{f}=0.34$ (hexanes/EtOAc, $2 / 1$ ); $[\alpha]_{\mathrm{D}}{ }^{20}=-4.5^{\circ}$ (c $\left.1, \mathrm{CHCl}_{3}\right)$; ${ }^{1} \mathrm{H}$ NMR (400 MHz, $\left.\mathrm{CDCl}_{3}\right) \delta 4.02-3.98(\mathrm{~m}$, $2 \mathrm{H}), 3.83(\mathrm{dd}, J=9.4,6.1 \mathrm{~Hz}, 1 \mathrm{H}), 3.76(\mathrm{dd}, J=6.8,4.6$ $\mathrm{Hz}, 1 \mathrm{H}), 3.47$ (dd, $J=11.3,6.9 \mathrm{~Hz}, 1 \mathrm{H}), 3.40$ (dd, $J=$ 11.3, 5.1 Hz, $1 \mathrm{H}), 3.16(\mathrm{dd}, J=6.8,5.3 \mathrm{~Hz}, 1 \mathrm{H}), 2.00$ (ddd, $J=12.3,8.5,3.7 \mathrm{~Hz}, 1 \mathrm{H}), 1.84-1.76(\mathrm{~m}, 2 \mathrm{H}), 1.63$ (dt, $J=12.3,8.7 \mathrm{~Hz}, 1 \mathrm{H}), 1.43$ (bs, $3 \mathrm{H}), 1.33$ (bs, $3 \mathrm{H}$ ), $1.17(\mathrm{~s}, 3 \mathrm{H}), 0.90(\mathrm{~s}, 3 \mathrm{H}), 0.86(\mathrm{~s}, 3 \mathrm{H}) ;{ }^{13} \mathrm{C}$ NMR (100 $\left.\mathrm{MHz}, \mathrm{CDCl}_{3}\right) \delta 109.4,86.5,83.3,80.1,71.7,65.7,37.3$, $33.8,27.1,26.2,24.8,22.9,22.1$; IR (neat) v 3458, 2965, 2873, 1457, 1370, 1261, 1211, 1156, 1058, 900, 854; HRMS (ESI) found 281.1738 [M + Na $]^{+}$, calcd 281.1729 for $\mathrm{C}_{19} \mathrm{H}_{32} \mathrm{O}_{3} \mathrm{Na}^{+}$.<smiles>COC(=O)[C@H](C)CSc1nnnn1-c1ccccc1</smiles>

(no compound number was assigned in the manuscript) (2S)-Methyl-3-(1-phenyl-1H-tetrazol-5-ylsulfanyl)propionic acid methyl ester. A flame-dried $100 \mathrm{~mL}$ round bottom flask equipped with a pressure equalized dropping funnel was charged with (-)-methyl D- $\beta$ hydroxyisobutyrate 10 (1 g, $8.47 \mathrm{mmol})$, triphenylphosphine $(3.33 \mathrm{~g}, 12.7 \mathrm{mmol})$ and $20 \mathrm{~mL}$ of anhydrous THF. The solution was cooled to $0^{\circ} \mathrm{C}$ and a solution of DIAD (2.57 g, $12.7 \mathrm{mmol})$ and 1-phenyl- $1 H$-tetrazole-5-thiol (2.26 g, $12.7 \mathrm{mmol})$ in $20 \mathrm{~mL}$ of anhydrous THF was added dropwise via the dropping funnel. After warming to room temperature within $2 \mathrm{~h}$ the solution was stirred for additional $3 \mathrm{~h}$. Then $40 \mathrm{~mL} \mathrm{H}_{2} \mathrm{O}$ and $100 \mathrm{~mL}$ MTBE were added. The aqueous layer was separated and extracted with $\operatorname{MTBE}(2 \times 50 \mathrm{~mL})$. The combined organic layers were dried over anhydrous $\mathrm{Na}_{2} \mathrm{SO}_{4}$ and concentrated under reduced pressure. Flash chromatography (hexanes/EtOAc, 
2.5:1) afforded the title compound, $2.31 \mathrm{~g}(8.29 \mathrm{mmol}$, $98 \%$ ), as an oil.

Data are: tlc $R_{f}=0.33$ (hexanes/EtOAc, 2.5:1); $[\alpha]_{\mathrm{D}}^{20}=$ 89.5 $5^{\circ}$ (c 1, $\left.\mathrm{CHCl}_{3}\right) ;{ }^{1} \mathrm{H}$ NMR $\left(400 \mathrm{MHz}, \mathrm{CDCl}_{3}\right) \delta$ 7.59$7.53(\mathrm{~m}, 5 \mathrm{H}), 3.70(\mathrm{~s}, 3 \mathrm{H}), 3.58(\mathrm{dm}, J=6.9 \mathrm{~Hz}, 2 \mathrm{H})$, 3.16-3.06 (m, $1 \mathrm{H}), 1.35(\mathrm{~d}, J=7.2 \mathrm{~Hz}, 3 \mathrm{H}) ;{ }^{13} \mathrm{C}$ NMR $\left(100 \mathrm{MHz}, \mathrm{CDCl}_{3}\right) \delta 174.7,154.0,133.4,130.0,129.7$, 123.6, 51.9, 39.4, 35.5, 16.8; IR (neat) v 3068, 2979, 2953, 2879, 2854, 1732, 1597, 1500, 1460, 1434, 1411, 1387, $1357,1302,1279,1239,1220,1171,1114,1090,1075$, $1042,1015,981,916,860,825,762,694 ;$ MS (EI) $\mathrm{m} / z(\%)$ $278\left(\mathrm{M}^{+}, 14\right), 204$ (29), 162 (39), 145 (50), 133 (39), 120 (32), 118 (71), 101 (17), 85 (14), 77 (20), 76 (100), 73 (19). HRMS (EI) found $278.0836 \mathrm{M}^{+}$, calcd 278.0838 for $\mathrm{C}_{12} \mathrm{H}_{14} \mathrm{~N}_{4} \mathrm{SO}_{2}$.

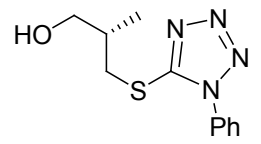

11

(2S)-Methyl-3-(1-phenyl-1H-tetrazol-5-ylsulfanyl)-

propan-1-ol 11. A $500 \mathrm{~mL}$ round bottom flask equipped with a pressure equalized dropping funnel was charged with the thioether (11.8 g, $42.4 \mathrm{mmol})$ described above and $150 \mathrm{~mL}$ of anhydrous THF. The solution was then cooled to $0^{\circ} \mathrm{C}$ and $\mathrm{LiEt}_{3} \mathrm{BH}(93 \mathrm{~mL}, 1.0 \mathrm{M} \mathrm{THF})$ was cautiously added during $1 \mathrm{~h}$ via dropping funnel. After an additional hour $70 \mathrm{~mL}$ of saturated $\mathrm{NH}_{4} \mathrm{Cl}$ solution was added. THF was distilled off under reduced pressure and the resulting mixture was extracted with $\mathrm{CH}_{2} \mathrm{Cl}_{2}(3 \times 50 \mathrm{~mL})$. The organic layer was dried over anhydrous $\mathrm{Na}_{2} \mathrm{SO}_{4}$ and concentrated. Flash chromatography (hexanes/EtOAc, 2.5:1) afforded the title compound, $9.91 \mathrm{~g}(39.6 \mathrm{mmol}$, $93 \%)$, as an oil.

Data are: tlc $R_{f}=0.09$ (hexanes/EtOAc, 2.5:1); $[\alpha]_{\mathrm{D}}{ }^{20}=$ $10.4^{\circ}$ (c 1, $\mathrm{CHCl}_{3}$ ); ${ }^{1} \mathrm{H}$ NMR $\left(400 \mathrm{MHz}, \mathrm{CDCl}_{3}\right) \delta 7.62-$ $7.52(\mathrm{~m}, 5 \mathrm{H}), 3.67(\mathrm{dd}, J=11.5,4.3 \mathrm{~Hz}, 2 \mathrm{H}), 3.55-3.44$ $(\mathrm{m}, 3 \mathrm{H}), 2.27-2.15(\mathrm{~m}, 1 \mathrm{H}), 1.06(\mathrm{~d}, J=6.9 \mathrm{~Hz}, 3 \mathrm{H}) ;{ }^{13} \mathrm{C}$ NMR $\left(100 \mathrm{MHz}, \mathrm{CDCl}_{3}\right) \delta 155.4,133.5,130.2,129.8$, $123.9,64.6,36.8,36.3,15.9$; IR (neat) $\vee 3391,3066,2960$, 2930, 2874, 2337, 1596, 1498, 1460, 1409, 1385, 1317, 1278, 1240, 1176, 1089, 1073, 1038, 1014, 984, 941, 915, 841, 814, 759, 713, 685, 552, 516; MS (EI) $\mathrm{m} / \mathrm{z}$ (\%) 250 $\left(\mathrm{M}^{+}, 6\right), 220$ (66), 208 (22), 203 (17), 179 (42), 178 (91), 163 (27), 150 (36), 135 (45), 119 (47), 118 (100), 117 (68), 104 (83), 91 (54), 77 (75), 65 (30); HRMS (EI) found $250.0894 \mathrm{M}^{+}$, calcd 250.0888 for $\mathrm{C}_{11} \mathrm{H}_{14} \mathrm{~N}_{4} \mathrm{SO}_{2}$.<smiles>C=C[C@H](C)CSc1nnnn1-c1ccccc1</smiles>

(no compound number was assigned in the manuscript) 5-[(2S)-Methyl-but-3-enylsulfanyl]-1-phenyl-1 H-

tetrazole. A $250 \mathrm{~mL}$ round bottom flask was charged with alcohol $11(2.28 \mathrm{~g}, 9.1 \mathrm{mmol})$ and $80 \mathrm{~mL}$ of anhydrous $\mathrm{CH}_{2} \mathrm{Cl}_{2}$. The solution was then cooled to $0^{\circ} \mathrm{C}$ and $\mathrm{NaHCO}_{3}$ (2.28 g, $27.3 \mathrm{mmol}$ ) was added, followed by Dess-Martin periodinane $(4.96 \mathrm{~g}, 11.7 \mathrm{mmol})$ in portions. After 30 minutes the mixture is poured in $100 \mathrm{~mL}$ of saturated $\mathrm{NaHCO}_{3}$ solution and diluted with $200 \mathrm{~mL}$ hexanes. The organic layer was washed twice with $\mathrm{NaHCO}_{3}$ solution $(100 \mathrm{~mL})$, and the combined aqueous layers were reextracted once with hexanes $(100 \mathrm{~mL})$. The combined hexane solutions were dried over $\mathrm{Na}_{2} \mathrm{SO}_{4}$, filtered, and concentrated to furnish the crude aldehyde. A flame-dried 3 -neck $250 \mathrm{~mL}$ round bottom flask equipped with a pressure equalized dropping funnel was charged with methyl triphenylphosphonium bromide $(6.5 \mathrm{~g}, 18.2 \mathrm{mmol})$ and $100 \mathrm{~mL}$ of anhydrous THF. This mixture was cooled to $0^{\circ} \mathrm{C}$ and LDA (9 mL, $2 \mathrm{M} \mathrm{THF} /$ heptane) was introduced via syringe in portions. After 30 minutes the crude aldehyde, dissolved in $25 \mathrm{~mL}$ of anhydrous THF, was added slowly via dropping funnel. After 30 minutes the reaction was terminated with $50 \mathrm{~mL}$ of saturated $\mathrm{NH}_{4} \mathrm{Cl}$ solution. THF was distilled of in vacuum and the resulting mixture was extracted with $\mathrm{CH}_{2} \mathrm{Cl}_{2}(3 \times 50 \mathrm{~mL})$. The organic layer was dried over anhydrous $\mathrm{Na}_{2} \mathrm{SO}_{4}$ and concentrated. Flash chromatography (hexanes/EtOAc, 10:1) delivers the desired olefin, $1.70 \mathrm{~g}(6.9 \mathrm{mmol}, 76 \%)$, as an oil.

Data are: tlc $R_{f}=0.50$ (hexanes/EtOAc, 2.5:1); $[\alpha]_{\mathrm{D}}^{20}=$ $-15.9^{\circ}\left(c 1, \mathrm{CHCl}_{3}\right) ;{ }^{1} \mathrm{H}$ NMR $\left(400 \mathrm{MHz}, \mathrm{CDCl}_{3}\right) \delta$ 7.637.49 (m, $5 \mathrm{H}), 5.75$ (ddd, $J=17.3,10.2,7.2 \mathrm{~Hz}, 1 \mathrm{H}), 5.12-$ $5.02(\mathrm{~m}, 2 \mathrm{H}), 3.42(\mathrm{~d}, J=2.5 \mathrm{~Hz}, 1 \mathrm{H}), 3.41(\mathrm{~d}, J=3.1$ $\mathrm{Hz}, 1 \mathrm{H}$ ), 2.68 (app sept, $J=7.0 \mathrm{~Hz}, 1 \mathrm{H}$ ), 1.17 (d, $J=6.8$ $\mathrm{Hz}, 3 \mathrm{H}) ;{ }^{13} \mathrm{C}$ NMR $\left(100 \mathrm{MHz}, \mathrm{CDCl}_{3}\right) \delta 154.5,140.9$, $133.8,130.1,129.8,123.9,115.3,39.5,37.4,19.3$; IR (neat) $\vee 3073,2966,2930,2870,2361,2338,1752,1737$, $1641,1597,1499,1459,1410,1386,1332,1316,1279$, $1238,1176,1159,1088,1075,1055,1041,1014,994,918$, $860,821,760,694,608,553,521,503$; MS (EI) $\mathrm{m} / z(\%)$ 246 (M+, 74), 231 (20), 203 (27), 199 (95), 185 (19), 178 (34), $176(53), 171$ (18), 163 (73), 150 (30), 145 (60), 135 (74), 117 (100), 104 (29), 101 (74), 91 (74), 85 (32), 77 (86), 69 (28), 67 (74), 65 (74); HRMS (EI) found 246.0934 $\mathrm{M}^{+}$, calcd 246.0939 for $\mathrm{C}_{12} \mathrm{H}_{14} \mathrm{~N}_{4} \mathrm{~S}$.

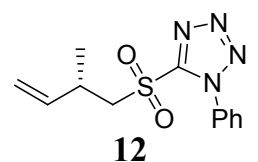

\section{5-[(2S)-methyl-but-3-ene-1-sulfonyl]-1-phenyl-1 $H$ -}

tetrazole 12. A $250 \mathrm{~mL}$ round bottom flask was charged with the olefin from the Wittig reaction $(2.81 \mathrm{~g}, 11.4$ $\mathrm{mmol}$ ) and $75 \mathrm{~mL}$ of EtOH. The solution was then cooled to $0^{\circ} \mathrm{C}$ and $\left(\mathrm{NH}_{4}\right)_{6} \mathrm{Moo}_{7} \mathrm{O}_{24} \cdot 4 \mathrm{H}_{2} \mathrm{O}(1.41 \mathrm{~g}, 1.14 \mathrm{mmol})$ in $35 \% \mathrm{H}_{2} \mathrm{O}_{2}(10 \mathrm{~mL}, 114 \mathrm{mmol})$ was added. The reaction was warmed to room temperature and stirred for $24 \mathrm{~h}$. Then $75 \mathrm{~mL} \mathrm{H}_{2} \mathrm{O}$ and $150 \mathrm{~mL} \mathrm{CH}_{2} \mathrm{Cl}_{2}$ were added and the layers were separated. The aqueous layer was extracted with $\mathrm{CH}_{2} \mathrm{Cl}_{2}(2 \times 100 \mathrm{~mL})$, and the combined organic solutions were dried over $\mathrm{Na}_{2} \mathrm{SO}_{4}$ and concentrated under reduced pressure. Flash chromatography (hexanes/EtOAc, 2.5:1) furnished the desired sulfon, $2.92 \mathrm{~g}(10.5 \mathrm{mmol}, 92 \%)$, as a colourless solid.

Data are: tlc $R_{f}=0.49$ (hexanes/EtOAc, $2.5: 1$ ); $\mathrm{mp}=62^{\circ} \mathrm{C}$; $[\alpha]_{\mathrm{D}}{ }^{20}=-19.4^{\circ}\left(c 1, \mathrm{CHCl}_{3}\right) ;{ }^{1} \mathrm{H}$ NMR $\left(400 \mathrm{MHz}, \mathrm{CDCl}_{3}\right) \delta$ 7.68-7.55 (m, 5 H), 5.74 (ddd, $J=17.3,10.3,7.5 \mathrm{~Hz}, 1 \mathrm{H}$ ), 
$5.09(\mathrm{dm}, J=17.3 \mathrm{~Hz}, 1 \mathrm{H}), 5.02(\mathrm{dm}, J=10.3 \mathrm{~Hz}, 1 \mathrm{H})$, $3.90(\mathrm{dd}, J=14.6,7.1 \mathrm{~Hz}, 1 \mathrm{H}), 3.68(\mathrm{dd}, J=14.6,6.4 \mathrm{~Hz}$, $1 \mathrm{H}), 3.02$ (app sep, $J=7.0 \mathrm{~Hz}, 1 \mathrm{H}), 1.24(\mathrm{~d}, J=6.9 \mathrm{~Hz}, 3$ $\mathrm{H}) ;{ }^{13} \mathrm{C}$ NMR $\left(100 \mathrm{MHz}, \mathrm{CDCl}_{3}\right) \delta 153.9,139.5,133.0$, 131.4, 129.6, 125.2, 115.7, 61.3, 32.7, 19.9; IR (neat) $\mathrm{v}$ 3081, 2971, 2934, 2873, 1643, 1595, 1497, 1460, 1422, 1399, 1347, 1295, 1255, 1205, 1152, 1098, 1075, 1045, 1015, 995, 921, 876, 808, 763, 690, 623, 574, 545, 523; MS (EI) $m / z(\%) 278\left(\mathrm{M}^{+}, 39\right), 145$ (55), 131 (30), 123 (12), 119 (35), 118 (100), 117 (68), 91 (37), 77 (55), 69 (72), 68 (29), 67 (20), 65 (55); HRMS (EI) found 278.0837 $\mathrm{M}^{+}$, calcd 278.0838 for $\mathrm{C}_{12} \mathrm{H}_{14} \mathrm{~N}_{4} \mathrm{SO}_{2}$; Anal. calcd for $\mathrm{C}_{12} \mathrm{H}_{14} \mathrm{~N}_{4} \mathrm{SO}_{2}$ : C, 51.78, H 5.07, N 20.13, found: C, 51.47, H 5.10, N 20.09 .

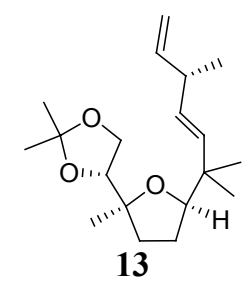

Julia product 13. A $100 \mathrm{~mL}$ round bottom flask was charged with alcohol 9 (1.64 g, $6.35 \mathrm{mmol})$ and $40 \mathrm{~mL}$ of dry $\mathrm{CH}_{2} \mathrm{Cl}_{2}$. Subsequently powdered $4 \AA$ molecular sieves (1.04 g), NMO (1.11 g, $9.52 \mathrm{mmol})$ and TPAP (112 mg, $0.318 \mathrm{mmol}$ ) were added at room temperature. After $1 \mathrm{~h}$, the reaction mixture was filtered over a plug of silica gel, washed, and the resulting filtrate was concentrated under reduced pressure. The obtained aldehyde (1.37 g, 5.35 mmol, 84\%) was directly subjected to the following olefination prodcedure without further purification. A flame-dried $100 \mathrm{~mL}$ round bottom flask was charged with sulfone 12 (1.93 g, $6.95 \mathrm{mmol})$ and $35 \mathrm{~mL}$ of dry THF. The solution was cooled to $-78^{\circ} \mathrm{C}$, then LDA (4 mL, $2 \mathrm{M}$ $\mathrm{THF} /$ heptane) was added dropwise via syringe. After 20 minutes, the aldehyde mentioned above (1.37 g, 5.35 $\mathrm{mmol}$ ) in $5 \mathrm{~mL}$ of dry THF was added. The solution was allowed to warm to room temperature and stirred for $16 \mathrm{~h}$, and was then refluxed over $4 \mathrm{~h}$ before cooling to room temperature. The reaction mixture was terminated with half-saturated $\mathrm{NH}_{4} \mathrm{Cl}$ solution $(20 \mathrm{~mL})$, and the aqueous layer was extracted with $\operatorname{MTBE}(3 \times 10 \mathrm{~mL})$. The combined organic layers were dried over anhydrous $\mathrm{Na}_{2} \mathrm{SO}_{4}$ and concentrated under reduced pressure. Flash chromatography (hexanes/EtOAc, 10:1) afforded the title compound, $1.50 \mathrm{~g}$ (4.86 mmol, $91 \%, 76 \%$ over 2 steps), as an oil.

Data are: tlc $R_{f}=0.46$ (hexanes/EtOAc $10 / 1$ ); $[\alpha]_{\mathrm{D}}{ }^{20}=$ $+25.4^{\circ}\left(c\right.$ 1, $\left.\mathrm{CHCl}_{3}\right) ;{ }^{1} \mathrm{H} \mathrm{NMR}\left(400 \mathrm{MHz}, \mathrm{CDCl}_{3}\right) \delta 5.79$ (ddd, $J=17.1,10.4,6.6 \mathrm{~Hz}, 1 \mathrm{H}), 5.43(\mathrm{dd}, J=15.9,0.9$ $\mathrm{Hz}, 1 \mathrm{H}), 5.31(\mathrm{dd}, J=15.9,6.5 \mathrm{~Hz}, 1 \mathrm{H}), 4.90-5.02$ (m, 2 H), 3.87-4.03 (m, 3 H), 3.65-3.70 (m, $1 \mathrm{H}), 2.77-2.87$ (m, 1 H), 1.85-1.97 (m, $1 \mathrm{H}), 1.72-1.80(\mathrm{~m}, 1 \mathrm{H}), 1.59-1.69(\mathrm{~m}, 2$ H), 1.42 (s, 3 H), 1.33 (s, 3 H), 1.16 (s, 3 H), 1.08 (d, J= $6.9 \mathrm{~Hz}, 3 \mathrm{H}), 0.99(\mathrm{~s}, 3 \mathrm{H}), 0.94(\mathrm{~s}, 3 \mathrm{H}) ;{ }^{13} \mathrm{C}$ NMR $(100$ $\left.\mathrm{MHz}, \mathrm{CDCl}_{3}\right) \delta 143.3,135.2,131.6,112.5,109.1,86.3$, $82.5,80.0,65.6,40.5,38.9,35.9,26.9,26.3,24.9,24.5$, $24.3,21.1,20.1$; IR (neat) $v 3081,2964,2930,2872,1784$, $1715,1635,1602,1494,1454,1380,1369,1262,1210$,
1155, 1069, 1019, 995, 979, 911, 855, 798, 747, 699; HRMS (ESI) found 331.2244 [M $+\mathrm{Na}^{+}$, calcd 331.2249 for $\mathrm{C}_{19} \mathrm{H}_{32} \mathrm{O}_{3} \mathrm{Na}^{+}$.

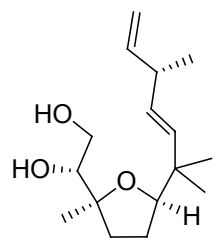

(no compound number was assigned in the manuscript) 1,2-Diol. A $100 \mathrm{~mL}$ round bottom flask was charged with Julia product $13(640 \mathrm{mg}, 2.07 \mathrm{mmol})$ and $30 \mathrm{~mL}$ of $\mathrm{MeOH}$. After addition of $p \mathrm{TsOH}(79 \mathrm{mg}, 0.415 \mathrm{mmol})$ the solution was warmed to $50^{\circ} \mathrm{C}$ for $18 \mathrm{~h}$ and then cooled to room temperature. The reaction was stopped by addition of saturated $\mathrm{NaHCO}_{3}$ solution $(20 \mathrm{~mL})$, and the mixture was extracted with $\mathrm{CH}_{2} \mathrm{Cl}_{2}(3 \times 30 \mathrm{~mL})$. The combined organic phases were dried over $\mathrm{Na}_{2} \mathrm{SO}_{4}$ and the crude product was purified over a short column (hexanes/EtOAc, 10:1 $\rightarrow$ EtOAc) to provide the desired diol, $405 \mathrm{mg}$ (1.51 mmol, $73 \%$ ), as an oil, along with recovered starting material, 168 $\mathrm{mg}(545 \mu \mathrm{mol},>26 \%)$.

Data are: tlc $R_{f}=0.04$ (hexanes/ EtOAc, $10: 1$ ); $[\alpha]_{\mathrm{D}}{ }^{20}=$ $+9.9^{\circ}\left(c 1, \mathrm{CHCl}_{3}\right) ;{ }^{1} \mathrm{H}$ NMR $\left(400 \mathrm{MHz}, \mathrm{CDCl}_{3}\right) \delta 5.78$ (ddd, $J=17.1,10.4,6.6 \mathrm{~Hz}, 1 \mathrm{H}), 5.44(\mathrm{~d}, J=15.9 \mathrm{~Hz}, 1$ H), $5.38(\mathrm{dd}, J=15.9,5.8 \mathrm{~Hz}, 1 \mathrm{H}), 5.03-4.91(\mathrm{~m} 2 \mathrm{H})$, 3.74-3.63 (m, $3 \mathrm{H}), 3.56$ (dd, $J=10.8,7.0 \mathrm{~Hz}, 1 \mathrm{H}), 2.89$ $2.79(\mathrm{~m}, 1 \mathrm{H}), 2.75$ (br s, $2 \mathrm{H}), 1.97-1.78$ (m, $2 \mathrm{H}), 1.70-$ $1.59(\mathrm{~m}, 1 \mathrm{H}), 1.55-1.46(\mathrm{~m}, 1 \mathrm{H}), 1.15(\mathrm{~s}, 3 \mathrm{H}), 1.09$ (d, $J$ $=6.9 \mathrm{~Hz}, 3 \mathrm{H}), 1.04(\mathrm{~s}, 3 \mathrm{H}), 0.96(\mathrm{~s}, 3 \mathrm{H}) ;{ }^{13} \mathrm{C} \mathrm{NMR}(100$ $\left.\mathrm{MHz}, \mathrm{CDCl}_{3}\right) \delta 143.1,134.4,132.7,112.7,84.9,84.1$, $76.6,63.2,40.4,38.9,32.5,26.9,24.6,24.5,22.1,20.0$; HRMS (ESI) found 291.1949 [M + Na] ${ }^{+}$, calcd 291.1936 for $\mathrm{C}_{16} \mathrm{H}_{28} \mathrm{O}_{3} \mathrm{Na}^{+}$.

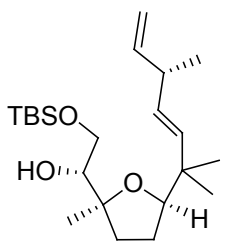

(no compound number was assigned in the manuscript) TBS-alcohol. A $100 \mathrm{~mL}$ round bottom flask was charged with the deprotected 1,2-diol (660 $\mathrm{mg}, 2.46 \mathrm{mmol})$ and 30 $\mathrm{mL}$ of dry $\mathrm{CH}_{2} \mathrm{Cl}_{2}$. Then imidazole $(330 \mathrm{mg}, 4.85 \mathrm{mmol})$ was added, followed by TBS-Cl $(580 \mathrm{mg}, 3.85 \mathrm{mmol})$ and DMAP (15 mg, $0.123 \mathrm{mmol}$ ). After 30 minutes the reaction was terminated by addition of saturated $\mathrm{NH}_{4} \mathrm{Cl}$ solution (10 $\mathrm{mL})$. The aqueous layer was extracted once with $\mathrm{CH}_{2} \mathrm{Cl}_{2}$ $(10 \mathrm{~mL})$, and the combined organic solutions were dried over $\mathrm{Na}_{2} \mathrm{SO}_{4}$ and concentrated under reduced pressure. Filtration over silica gel (hexanes/EtOAc, 10:1) afforded the TBS-protected alcohol, $929 \mathrm{mg}$ (2.43 $\mathrm{mmol}, 99 \%)$, as an oil.

Data are: tlc $R_{f}=0.49$ (hexanes/EtOAc, 10:1); $[\alpha]_{\mathrm{D}}{ }^{20}=$ $+21.0^{\circ}\left(c 1, \mathrm{CHCl}_{3}\right) ;{ }^{1} \mathrm{H} \mathrm{NMR}\left(400 \mathrm{MHz}, \mathrm{CDCl}_{3}\right) \delta 5.78$ (ddd, $J=17.1,10.4,6.5 \mathrm{~Hz}, 1 \mathrm{H}), 5.43$ (dd, $J=15.9,0.6$ Hz, $1 \mathrm{H}$ ), 5.33 (dd, $J=15.9,6.5 \mathrm{~Hz}, 1 \mathrm{H}$ ), 5.00-4.90 (m 2 
H), $3.76(\mathrm{dd}, J=10.3 \mathrm{~Hz}, 3.9 \mathrm{~Hz}, 1 \mathrm{H}), 3.69$ (dd, $J=8.0$, $6.7 \mathrm{~Hz}, 1 \mathrm{H}), 3.62$ (dd, $J=10.3,7.0 \mathrm{~Hz}, 1 \mathrm{H}), 3.51$ (ddd, $J$ $=7.0,3.9,2.5 \mathrm{~Hz}, 1 \mathrm{H}), 2.82(\operatorname{app} \operatorname{sex}, J=6.5 \mathrm{~Hz}, 1 \mathrm{H})$, $2.64(\mathrm{~d}, J=2.5 \mathrm{~Hz}, 1 \mathrm{H}), 2.02-1.94(\mathrm{~m}, 1 \mathrm{H}), 1.84-1.75(\mathrm{~m}$, $1 \mathrm{H}), 1.67-1.50(\mathrm{~m}, 2 \mathrm{H}), 1.14(\mathrm{~s}, 3 \mathrm{H}), 1.07$ (d, $J=6.9 \mathrm{~Hz}$, $3 \mathrm{H}), 1.01(\mathrm{~s}, 3 \mathrm{H}), 0.95(\mathrm{~s}, 3 \mathrm{H}), 0.90(\mathrm{~s}, 9 \mathrm{H}), 0.08(\mathrm{~s}, 6$ $\mathrm{H}) ;{ }^{13} \mathrm{C} \mathrm{NMR}\left(100 \mathrm{MHz}, \mathrm{CDCl}_{3}\right) \delta 143.2,135.0,132.1$, $112.6,85.5,83.7,76.6,63.7,40.4,39.0,34.2,26.9,25.9$, $24.5,24.3,21.7,20.1,18.3,-5.36,-5.37$; IR (neat) v 3575, 3080, 2958, 2930, 2858, 2711, 1636, 1463, 1408, 1385, 1362, 1327, 1253, 1096, 1061, 1024, 994, 938, 910, 834, 776, 741, 677; HRMS (ESI) found 405.2817 [M $+\mathrm{Na}^{+}$, calcd 408.2801 for $\mathrm{C}_{22} \mathrm{H}_{42} \mathrm{O}_{3} \mathrm{SiNa}^{+}$.

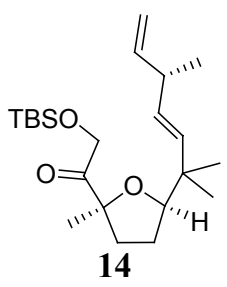

TBS-ketone 14. A $100 \mathrm{~mL}$ round bottom flask was charged with the TBS protected alcohol $(875 \mathrm{mg}, 2.29$ mmol) and $20 \mathrm{~mL}$ of dry $\mathrm{CH}_{2} \mathrm{Cl}_{2}$. The solution was cooled to $0^{\circ} \mathrm{C}$, then Dess-Martin periodinane $(1.5 \mathrm{~g}, 3.54 \mathrm{mmol})$ was added. After addition the reaction mixture was warmed to room temperature and stirred for $2 \mathrm{~h}$, before saturated $\mathrm{NaHCO}_{3}$ solution $(20 \mathrm{~mL})$ and $\mathrm{Na}_{2} \mathrm{~S}_{2} \mathrm{O}_{3}$ solution $(10 \mathrm{~mL})$ were added. The aqueous layer was separated and extracted once with $\mathrm{CH}_{2} \mathrm{Cl}_{2}(10 \mathrm{~mL})$. The combined organic solutions were dried over $\mathrm{Na}_{2} \mathrm{SO}_{4}$ and concentrated under reduced pressure. Flash chromatography (hexanes/EtOAc, 40:1) afforded the desired ketone, $828 \mathrm{mg}$ (2.17 $\mathrm{mmol}$, 95\%), as an oil.

Data are: tlc $R_{f}=0.53$ (hexanes/EtOAc, $\left.10: 1\right) ;[\alpha]_{\mathrm{D}}{ }^{20}=-$ $\left.12.3^{\circ}(c), \mathrm{CHCl}_{3}\right) ;{ }^{1} \mathrm{H} \mathrm{NMR}\left(400 \mathrm{MHz}, \mathrm{CDCl}_{3}\right) \delta 5.77$ (ddd, $J=17.1,10.4,6.6 \mathrm{~Hz}, 1 \mathrm{H}), 5.42(\mathrm{~d}, J=16.0 \mathrm{~Hz}, 1$ H), $5.34(\mathrm{dd}, J=16.0, J=6.6 \mathrm{~Hz}, 1 \mathrm{H}), 5.01-4.90(\mathrm{~m} 2 \mathrm{H})$, 4.69 (br s, $2 \mathrm{H}), 3.75$ (dd, $J=8.2,6.1 \mathrm{~Hz}, 1 \mathrm{H}), 2.82$ (ps sex, $J=6.6 \mathrm{~Hz}, 1 \mathrm{H}), 2.03-1.98(\mathrm{~m}, 1 \mathrm{H}), 1.85-1.72(\mathrm{~m}$, $2 \mathrm{H}), 1.58-1.49$ (m, $1 \mathrm{H}), 1.29$ (s, $3 \mathrm{H}), 1.07$ (d, $J=6.9 \mathrm{~Hz}$, $3 \mathrm{H}), 1.03$ (s, $3 \mathrm{H}), 0.99$ (s, $3 \mathrm{H}), 0.92$ (s, $9 \mathrm{H}), 0.09$ (s, 3 $\mathrm{H}), 0.08(\mathrm{~s}, 3 \mathrm{H}) ;{ }^{13} \mathrm{C} \mathrm{NMR}\left(100 \mathrm{MHz}, \mathrm{CDCl}_{3}\right) \delta 211.6$, $143.1,134.7,132.2,112.7,87.8,86.8,66.5,40.5,38.8$, $35.8,26.4,25.8,24.3,24.2,23.8,20.1,18.6,-5.4,-5.5$; IR (neat) $v$ 3081, 2958, 2929, 2857, 1736, 1636, 1463, 1421, 1387, 1363, 1253, 1160, 1100, 1047, 1002, 938, 912, 835, 777, 735, 681; HRMS (ESI) found $387.2907[\mathrm{M}+\mathrm{Li}]^{+}$, calcd 387.2902 for $\mathrm{C}_{22} \mathrm{H}_{40} \mathrm{O}_{3} \mathrm{SiLi}^{+}$.

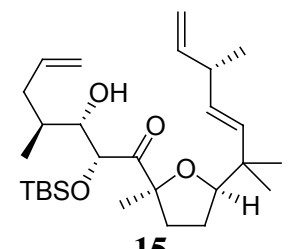

15

Aldol product 15. A $100 \mathrm{~mL}$ round bottom flask was charged with the alcohol precursor from aldehyde $\mathbf{B}^{6}$ (700 $\mathrm{mg}, 7.13 \mathrm{mmol}$ ) and $40 \mathrm{~mL}$ of dry $\mathrm{CH}_{2} \mathrm{Cl}_{2}$. The solution was cooled to $0^{\circ} \mathrm{C}$ and Dess-Martin periodinane $(3.56 \mathrm{~g}$, $8.39 \mathrm{mmol}$ ) was added. After 15 minutes the mixture was warmed to room temperature. After $1 \mathrm{~h}$ an additional small amount Dess-Martin periodinane was added, and after $2 \mathrm{~h}$ the reaction was terminated by addition of an aqueous $10 \%$ $\mathrm{Na}_{2} \mathrm{~S}_{2} \mathrm{O}_{3} /$ saturated $\mathrm{NaHCO}_{3}$ solution $(50 \mathrm{~mL})$. The organic layer was separated and successively washed with this solution $(4 \times 50 \mathrm{~mL})$. The combined aqueous phases were then reextracted once with $\mathrm{CH}_{2} \mathrm{Cl}_{2}(25 \mathrm{~mL})$, and the combined organic solutions were dried over $\mathrm{MgSO}_{4}$ and concentrated cautiously under reduced pressure $(p=600$ mbar). The volatile aldehyde obtained was directly subjected to the following aldol prodcedure. A $100 \mathrm{~mL}$ round bottom flask was charged with ketone 14 (650 mg, $1.71 \mathrm{mmol}$ ) and $40 \mathrm{~mL}$ of dry THF. The solution was cooled to $-78^{\circ} \mathrm{C}$, before $\mathrm{KHMDS}(3.42 \mathrm{~mL}, 0.5 \mathrm{M}$ toluene) was introduced dropwise. After 30 minutes the aldehyde $\mathbf{B}$ in dry THF $(5 \mathrm{~mL})$ was added and the solution was stirred for $2 \mathrm{~h}$. The reaction was then terminated by careful addition of $\mathrm{MeOH}$, followed by saturated $\mathrm{NH}_{4} \mathrm{Cl}$ solution $(10 \mathrm{~mL})$. The flask was warmed to room temperature in the cold bath, then the aqueous layer was separated and extracted with MTBE (3x $10 \mathrm{~mL})$. The combined organic solutions were dried over $\mathrm{Na}_{2} \mathrm{SO}_{4}$ and concentrated under reduced pressure. Flash chromatography (hexanes/EtOAc, 40:1) furnished the desired aldol product, $807 \mathrm{mg}$ (1.69 mmol, 99\%), as an oil.

Data are: tlc $R_{f}=0.42$ (hexanes/EtOAc, $10: 1$ ); $[\alpha]_{\mathrm{D}}{ }^{20}=$ $43.6^{\circ}$ (c 1, $\mathrm{CHCl}_{3}$ ); ${ }^{1} \mathrm{H}$ NMR $\left(400 \mathrm{MHz}, \mathrm{CDCl}_{3}\right) \delta$ 5.87$5.74(\mathrm{~m}, 1 \mathrm{H}), 5.76(\mathrm{ddd}, J=17.1,10.4,6.5 \mathrm{~Hz}, 1 \mathrm{H}), 5.44$ $(\mathrm{d}, J=16.1 \mathrm{~Hz}, 1 \mathrm{H}), 5.35(\mathrm{dd}, J=16.1,6.7 \mathrm{~Hz}, 1 \mathrm{H}), 5.08$ (br s, $1 \mathrm{H}), 5.08-4.89(\mathrm{~m}, 4 \mathrm{H}), 3.88$ (t, $J=10.6 \mathrm{~Hz}, 1 \mathrm{H})$, $3.79(\mathrm{dd}, J=7.8,6.8 \mathrm{~Hz}, 1 \mathrm{H}), 2.80($ app hex, $J=6.7 \mathrm{~Hz}, 1$ H), 2.61-2.52 (m, 1 H), 2.00-1.69 (m, $6 \mathrm{H}), 1.56-1.45$ (m, 1 H), 1.34 (s, $3 \mathrm{H}), 1.06(\mathrm{~d}, J=6.8 \mathrm{~Hz}, 3 \mathrm{H}), 1.03(\mathrm{~s}, 3 \mathrm{H})$, $1.00(\mathrm{~s}, 3 \mathrm{H}), 0.96$ (d, $J=6.8 \mathrm{~Hz}, 3 \mathrm{H}), 0.92$ (s, $9 \mathrm{H}), 0.10$ $(\mathrm{s}, 3 \mathrm{H}),-0.03(\mathrm{~s}, 3 \mathrm{H}) ;{ }^{13} \mathrm{C} \mathrm{NMR}\left(100 \mathrm{MHz}, \mathrm{CDCl}_{3}\right) \delta$ 212.7, 143.1, 137.2, 134.8, 132.2, 116.1, 112.7, 88.6, 86.3, $76.2,75.0,40.5,38.8,37.27,37.26,36.2,25.9,25.6,24.2$, $24.05,24.03,20.1,18.5,15.9,-4.2,-5.3$; IR (neat) v 3572, $3077,2959,2930,2858,1727,1638,1462,1387,1363$, 1253, 1214, 1160, 1100, 1043, 1022, 999, 967, 909, 860, 836, 807, 777, 672; HRMS (ESI) found 479.3577 [M + $\mathrm{H}]^{+}$, calcd 479.3557 for $\mathrm{C}_{28} \mathrm{H}_{50} \mathrm{O}_{4} \mathrm{SiH}^{+}$.

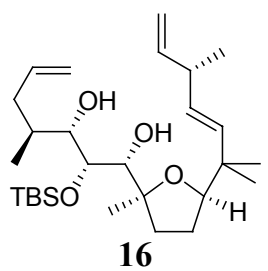

Syn-diol 16. A $25 \mathrm{~mL}$ round bottom flask was charged with aldol product $15(200 \mathrm{mg}, 418 \mu \mathrm{mol})$ and $10 \mathrm{~mL}$ of anhydrous $\mathrm{CH}_{2} \mathrm{Cl}_{2}$ and cooled to $-78^{\circ} \mathrm{C}$, before DIBAL-H (2.1 mL, $1 \mathrm{M}$ hexane) was added. Additional DIBAL-H $(0.8 \mathrm{~mL})$ was added after $2.5 \mathrm{~h}$, and a final portion $(0.5$ mL) 30 minutes thereafter. After $4.5 \mathrm{~h}$ the reaction mixture was allowed to warm to $-30^{\circ} \mathrm{C}$ and was then terminated with $\mathrm{H}_{2} \mathrm{O}(340 \mu \mathrm{L}) .340 \mu \mathrm{L}$ of a $4 \mathrm{M} \mathrm{NaOH}$ solution was 
introduced after reaching room temperature, followed by $\mathrm{H}_{2} \mathrm{O}(680 \mu \mathrm{L})$. After 15 minutes $\mathrm{Na}_{2} \mathrm{SO}_{4}$ was added and the mixture was filtered. The residue was washed and the combined organic solutions were concentrated under reduced pressure. Flash chromatography delivered the diol, $140 \mathrm{mg}(291 \mu \mathrm{mol}, 70 \%)$, as an oil, along with recovered starting material (56 mg, $117 \mu \mathrm{mol}, 28 \%$ ).

Data are: tlc $R_{f}=0.45$ (hexanes/EtOAc, $10: 1$ ); $[\alpha]_{\mathrm{D}}{ }^{20}=$ $+27.7^{\circ}\left(\mathrm{c} 1, \mathrm{CHCl}_{3}\right)$; ${ }^{1} \mathrm{H} \mathrm{NMR}\left(400 \mathrm{MHz}, \mathrm{CDCl}_{3}\right) \delta 5.86-$ $5.72(\mathrm{~m}, 1 \mathrm{H}), 5.77$ (ddd, $J=17.1,10.4,6.7 \mathrm{~Hz}, 1 \mathrm{H}), 5.43$ $(\mathrm{d}, J=15.9,1 \mathrm{H}), 5.37(\mathrm{dd}, J=15.9,5.9 \mathrm{~Hz}, 1 \mathrm{H}), 5.08-$ $4.89(\mathrm{~m}, 4 \mathrm{H}), 3.78(\mathrm{~d}, J=6.5 \mathrm{~Hz}, 1 \mathrm{H}), 3.71(\mathrm{dd}, J=8.7$, $6.2 \mathrm{~Hz}, 1 \mathrm{H}), 3.46$ (dd, $J=4.5$ u. $6.5 \mathrm{~Hz}, 1 \mathrm{H}), 3.17$ (t, $J=$ $8.6 \mathrm{~Hz}, 1 \mathrm{H}), 2.88(\mathrm{~d}, J=8.6 \mathrm{~Hz}, 1 \mathrm{H}), 2.84$ (app hex, $J=$ $6.3 \mathrm{~Hz}, 1 \mathrm{H}), 2.73(\mathrm{~d}, J=4.5 \mathrm{~Hz}, 1 \mathrm{H}), 2.53-2.44(\mathrm{~m}, 1 \mathrm{H})$, 2.14-2.06 (m, $1 \mathrm{H}), 1.97$ (dt, $J=13.7,8.3 \mathrm{~Hz}, 1 \mathrm{H}), 1.87-$ $1.74(\mathrm{~m}, 2 \mathrm{H}), 1.72-1.55(\mathrm{~m}, 2 \mathrm{H}), 1.21(\mathrm{~s}, 3 \mathrm{H}), 1.08$ (d, $J$ $=6.9 \mathrm{~Hz}, 3 \mathrm{H}), 1.03(\mathrm{~s}, 3 \mathrm{H}), 0.95(\mathrm{~s}, 3 \mathrm{H}), 0.90(\mathrm{~s}, 9 \mathrm{H})$, $0.83(\mathrm{~d}, J=6.8 \mathrm{~Hz}, 3 \mathrm{H}), 0.15(\mathrm{~s}, 3 \mathrm{H}), 0.11(\mathrm{~s}, 3 \mathrm{H}) ;{ }^{13} \mathrm{C}$ NMR $\left(100 \mathrm{MHz}, \mathrm{CDCl}_{3}\right) \delta 143.1,137.2,134.4,132.8$, $116.1,112.7,85.5,83.9,77.3,73.7,73.6,40.4,38.7,37.1$, $35.6,35.3,27.3,26.2,24.6,24.5,21.5,20.0,18.6,15.8$, $3.5,-4.76$; IR (neat) v 3551, 3077, 2959, 2930, 2857, 1639, 1462, 1386, 1362, 1329, 1249, 1215, 1116, 1071, 1037, 994, 959, 937, 910, 892, 858, 834, 802, 776, 681; HRMS (ESI) found $481.3733[\mathrm{M}+\mathrm{H}]^{+}$, calcd 481.3713 for $\mathrm{C}_{28} \mathrm{H}_{53} \mathrm{O}_{4} \mathrm{SiH}^{+}$.

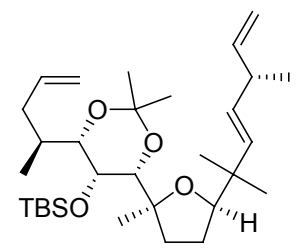

(no compound number was assigned in the manuscript)

1,3-Acetonide. A $25 \mathrm{~mL}$ round bottom flask was charged with the 1,3-diol $16(56 \mathrm{mg}, 117 \mu \mathrm{mol})$ and $3 \mathrm{~mL}$ of 2,2dimethoxy propane. $p \mathrm{TsOH}(8.5 \mathrm{mg}, 44.7 \mu \mathrm{mol})$ was added, and after $4 \mathrm{~h}$ the reaction was stopped by addition of $3 \mathrm{~mL}$ of saturated $\mathrm{NaHCO}_{3}$ solution. The solution was transferred to a separating funnel and extracted twice with $\mathrm{CH}_{2} \mathrm{Cl}_{2}(5 \mathrm{~mL})$. The organic phase was dried over $\mathrm{Na}_{2} \mathrm{SO}_{4}$ and concentrated under reduced pressure. A short filtration over silica gel (hexanes/EtOAc, 40:1) afforded the desired product, $60 \mathrm{mg}(115 \mu \mathrm{mol}, 99 \%)$, as an oil.

Data are: tlc $R_{f}=0.70$ (hexanes/EtOAc, $10: 1$ ); $[\alpha]_{\mathrm{D}}{ }^{20}=$ $\left.+7.5^{\circ}(c) 1, \mathrm{CHCl}_{3}\right) ;{ }^{1} \mathrm{H} \mathrm{NMR}\left(400 \mathrm{MHz}, \mathrm{CDCl}_{3}\right) \delta$ 5.79$5.69(\mathrm{~m}, 1 \mathrm{H}), 5.78(\mathrm{ddd}, J=17.1,10.3,6.5 \mathrm{~Hz}, 1 \mathrm{H}), 5.41$ $(\mathrm{dd}, J=15.9,0.6 \mathrm{~Hz}, 1 \mathrm{H}), 5.30(\mathrm{dd}, J=15.9,6.5 \mathrm{~Hz}, 1 \mathrm{H})$, $5.05-4.91(\mathrm{~m}, 4 \mathrm{H}), 3.78(\mathrm{dd}, J=8.3,4.6 \mathrm{~Hz}, 1 \mathrm{H}), 3.74(\mathrm{~s}$, $1 \mathrm{H}), 3.52$ (s, $1 \mathrm{H}), 3.18(\mathrm{~d}, J=9.7 \mathrm{~Hz}, 1 \mathrm{H}), 2.82$ (ps hex, $J=6.5 \mathrm{~Hz}, 1 \mathrm{H}), 2.37-2.27(\mathrm{~m}, 1 \mathrm{H}), 1.98-1.85(\mathrm{~m}, 3 \mathrm{H})$, 1.72-1.61 (m, $1 \mathrm{H}), 1.60-1.54(\mathrm{~m}, 2 \mathrm{H}), 1.46(\mathrm{~s}, 3 \mathrm{H}), 1.39$ $(\mathrm{s}, 3 \mathrm{H}), 1.22(\mathrm{~s}, 3 \mathrm{H}), 1.07(\mathrm{~d}, J=6.9 \mathrm{~Hz}, 3 \mathrm{H}), 0.99(\mathrm{~s}, 3$ H), 0.94 (s, $3 \mathrm{H}), 0.91(\mathrm{~s}, 9 \mathrm{H}), 0.81(\mathrm{~d}, J=6.5 \mathrm{~Hz}, 3 \mathrm{H})$, $0.10(\mathrm{~s}, 6 \mathrm{H}) ;{ }^{13} \mathrm{C}$ NMR $\left(100 \mathrm{MHz}, \mathrm{CDCl}_{3}\right) \delta 143.3,136.7$, $135.6,131.5,116.2,112.5,99.1,85.1,84.4,80.7,78.3$, $66.0,40.5,39.6,36.3,33.8,31.4,29.5,26.4,26.3,24.8$, $23.8,21.2,20.2,19.1,18.9,15.3,-1.6,-2.3$; IR (neat) $\mathrm{v}$ 3077, 2961, 2930, 2892, 2857, 2708, 1826, 1638, 1462,
$1414,1377,1305,1252,1198,1159,1101,1058,1029$, 979, 936, 909, 860, 834, 804, 771, 725, 696, 675; HRMS (ESI) found $521.4025[\mathrm{M}+\mathrm{H}]^{+}$, calcd 521.4026 for $\mathrm{C}_{31} \mathrm{H}_{56} \mathrm{O}_{4} \mathrm{SiH}^{+}$.

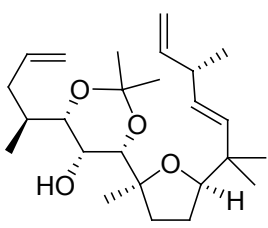

(no compound number was assigned in the manuscript)

2-deprotected 1,3-Acetonide. A $25 \mathrm{~mL}$ round bottom flask was charged with the TBS-protected acetonide (59 $\mathrm{mg}, 113 \mu \mathrm{mol})$ and $1 \mathrm{~mL}$ of anhydrous THF. TBAF (158 $\mathrm{mg}, 501 \mu \mathrm{mol})$ in $3 \mathrm{~mL}$ of anhydrous THF was added, and after $16 \mathrm{~h}$ the reaction was stopped by addition of $3 \mathrm{~mL}$ of saturated $\mathrm{NH}_{4} \mathrm{Cl}$ solution. The solution was directly filtered through a short column charged with silica gel (hexanes/EtOAc, 40:1) to afford the desired alcohol, $46 \mathrm{mg}$ $(113 \mu \mathrm{mol},>99 \%)$, as an oil.

Data are: tlc $R_{f}=0.48$ (hexanes/EtOAc, $10: 1$ ); $[\alpha]_{\mathrm{D}}{ }^{20}=$ $+21.7^{\circ}\left(\mathrm{c} 1, \mathrm{CHCl}_{3}\right) ;{ }^{1} \mathrm{H} \mathrm{NMR}\left(400 \mathrm{MHz}, \mathrm{CDCl}_{3}\right) \delta 5.84-$ $5.74(\mathrm{~m}, 1 \mathrm{H}), 5.78(\mathrm{ddd}, J=17.0,10.5$ u. $6.9 \mathrm{~Hz}, 1 \mathrm{H})$, $5.49(\mathrm{dd}, J=15.9,0.6 \mathrm{~Hz}, 1 \mathrm{H}), 5.28(\mathrm{dd}, J=15.9,6.9 \mathrm{~Hz}$, $1 \mathrm{H}), 5.04-4.87$ (m, $4 \mathrm{H}), 4.09$ (d, $J=2.6 \mathrm{~Hz}, 1 \mathrm{H}), 3.80$ $3.76(\mathrm{~m}, 1 \mathrm{H}), 3.71(\mathrm{dd}, J=8.0 \mathrm{u} .7 .1 \mathrm{~Hz}, 1 \mathrm{H}), 3.47(\mathrm{~s}, 1$ H), $3.24(\mathrm{~d}, J=9.5 \mathrm{~Hz}, 1 \mathrm{H}), 2.81(\operatorname{app}$ hex, $J=7.0 \mathrm{~Hz}, 1$ H), 2.42-2.33 (m, 1 H), 2.14-1.90 (m, 3 H), 1.76-1.65 (m, 2 $\mathrm{H}), 1.57-1.48(\mathrm{~m}, 1 \mathrm{H}), 1.45(\mathrm{~s}, 3 \mathrm{H}), 1.36(\mathrm{~s}, 3 \mathrm{H}), 1.26(\mathrm{~s}$, $3 \mathrm{H}), 1.05$ (d, $J=6.9 \mathrm{~Hz}, 3 \mathrm{H}), 1.00$ (s, $3 \mathrm{H}), 0.99$ (s, $3 \mathrm{H})$, $0.91(\mathrm{~d}, J=6.8 \mathrm{~Hz}, 3 \mathrm{H}) ;{ }^{13} \mathrm{C} \mathrm{NMR}\left(100 \mathrm{MHz}, \mathrm{CDCl}_{3}\right) \delta$ $143.5,136.8,136.1,131.2,116.4,112.3,98.8,86.8,84.7$, $76.5,75.6,63.7,40.5,39.1,36.8,35.2,32.4,29.7,26.9$, 24.7, 23.7, 23.2, 20.2, 18.9, 14.3; IR (neat) v 3478, 3076, 2968, 2934, 2872, 1729, 1638, 1462, 1377, 1262, 1202, $1175,1156,1120,1086,1062,1025,993,982,910,863$, 814; HRMS (ESI) found 429.2985 [M $+\mathrm{Na}]^{+}$, calcd 429.2981 for $\mathrm{C}_{25} \mathrm{H}_{42} \mathrm{O}_{4} \mathrm{Na}^{+}$.

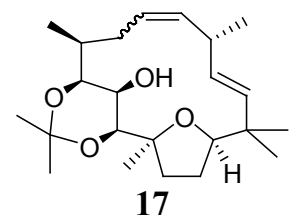

Metathesis product 17. A $50 \mathrm{~mL}$ round bottom flask with a reflux condenser was charged with the deprotected alcohol $(22 \mathrm{mg}, 54.1 \mu \mathrm{mol})$ and $27 \mathrm{~mL}$ of anhydrous $\mathrm{CH}_{2} \mathrm{Cl}_{2}$. Then Grubbs II catalyst $(4.6 \mathrm{mg}, 5.42 \mu \mathrm{mol})$ was added and the reaction was refluxed over $2 \mathrm{~h}$ before cooling to room temperature. The solution was saturated with air and silica gel $(100 \mathrm{mg})$ was added. After concentration the resulting solid was purified by flash column chromatography (hexanes/EtOAc, 10:1) to afford the macrocycle 17, $14.5 \mathrm{mg}(38.3 \mu \mathrm{mol}, 71 \%)$, as a colourless $\operatorname{solid}(Z: E \approx$ $6: 1)$.

Data are: tlc $R_{f}=0.43$ (hexanes/EtOAc, $\left.10: 1\right)$; $\mathrm{mp}=138^{\circ} \mathrm{C}$; $[\alpha]_{\mathrm{D}}{ }^{20}=+37.2^{\circ}\left(c 1, \mathrm{CHCl}_{3}\right) ;{ }^{1} \mathrm{H} \mathrm{NMR}\left(500 \mathrm{MHz}, \mathrm{CDCl}_{3}\right)$ $\delta$ (Z-isomer) $5.60(\mathrm{~d}, J=15.6 \mathrm{~Hz}, 1 \mathrm{H}), 5.28-5.17(\mathrm{~m}, 3 \mathrm{H})$, 
$3.92(\mathrm{~d}, J=11.3 \mathrm{~Hz}, 1 \mathrm{H}), 3.69(\mathrm{~d}, J=6.1 \mathrm{~Hz}, 1 \mathrm{H}), 3.54$ (dd, $J=11.8,4.4 \mathrm{~Hz}, 1 \mathrm{H}), 3.28$ (br s, $1 \mathrm{H}), 3.34-3.23(\mathrm{~m}, 1$ H), 2.55-2.42 (m, 2 H), 2.12-2.06 (m, $1 \mathrm{H}), 2.06-1.91(\mathrm{~m}, 2$ H), 1.86-1.77 (m, $1 \mathrm{H}), 1.70-1.62(\mathrm{~m}, 1 \mathrm{H}), 1.44(\mathrm{~s}, 3 \mathrm{H})$, $1.39(\mathrm{~s}, 3 \mathrm{H}), 1.35-1.27(\mathrm{~m}, 1 \mathrm{H}), 1.15(\mathrm{~s}, 3 \mathrm{H}), 1.11(\mathrm{~s}, 3$ H), 1.09 (d, $J=7.0 \mathrm{~Hz}, 3 \mathrm{H}), 1.01(\mathrm{~d}, J=6.6 \mathrm{~Hz}, 3 \mathrm{H}), 0.90$ (s, $3 \mathrm{H}$ ); $\delta$ (E-isomer) 5.72 (ddd, $J=15.1,11.0,3.2 \mathrm{~Hz}, 1$ H), 5.59 (d, $J=15.9 \mathrm{~Hz}, 1 \mathrm{H}), 5.38$ (ddd, $J=15.1,9.6,2.2$ $\mathrm{Hz}, 1 \mathrm{H}), 5.36(\mathrm{dd}, J=15.9,6.7 \mathrm{~Hz}, 1 \mathrm{H}), 4.17(\mathrm{~d}, J=10.4$ $\mathrm{Hz}, 1 \mathrm{H}), 3.65(\mathrm{~d}, J=9.9 \mathrm{~Hz}, 1 \mathrm{H}), 3.50(\mathrm{dd}, J=11.2,5.0$ $\mathrm{Hz}, 1 \mathrm{H}), 3.44($ br s, $1 \mathrm{H}), 2.92-2.83(\mathrm{~m}, 1 \mathrm{H}), 2.55-2.42$ (m, $2 \mathrm{H}), 2.12-2.06(\mathrm{~m}, 1 \mathrm{H}), 2.06-1.91(\mathrm{~m}, 2 \mathrm{H}), 1.86-1.77$ $(\mathrm{m}, 1 \mathrm{H}), 1.70-1.62(\mathrm{~m}, 1 \mathrm{H}), 1.41(\mathrm{~s}, 3 \mathrm{H}), 1.39(\mathrm{~s}, 3 \mathrm{H})$, 1.35-1.27 (m, $1 \mathrm{H}), 1.20(\mathrm{~d}, J=7.4 \mathrm{~Hz}, 3 \mathrm{H}), 1.15(\mathrm{~s}, 3 \mathrm{H})$, $1.11(\mathrm{~s}, 3 \mathrm{H}), 1.04(\mathrm{~d}, J=7.3 \mathrm{~Hz}, 3 \mathrm{H}), 0.91(\mathrm{~s}, 3 \mathrm{H}) ;{ }^{13} \mathrm{C}$ NMR (125 MHz, $\left.\mathrm{CDCl}_{3}\right) \delta$ (Z-isomer) 134.7, 132.8, 132.3, $125.7,99.5,86.4,82.3,77.7,74.3,63.6,38.2,36.7,35.9$, $33.1,31.7,29.9,28.1,28.0,26.2,25.9,21.3,19.1,16.2 ; \delta$ (E-isomer) $135.5,133.3,132.8,125.1,99.4,86.3,82.0$, 77.8, 77.4, 65.6, 41.4, 38.1, 36.4, 36.0, 32.7, 29.9, 28.2, 28.0, 27.0, 25.2, 21.4, 19.1, 13.4; IR (neat) v 3582, 2962, 2936, 2871, 2840, 1728, 1679, 1602, 1578, 1510, 1453, $1397,1381,1360,1304,1258,1201,1170,1101,1086$, 1071, 1056, 1035, 1000, 985, 907, 865, 835, 810, 732, 703; HRMS (ESI) found $379.2841[\mathrm{M}+\mathrm{H}]^{+}$, calcd 379.2848 for $\mathrm{C}_{23} \mathrm{H}_{39} \mathrm{O}_{4}^{+}$.

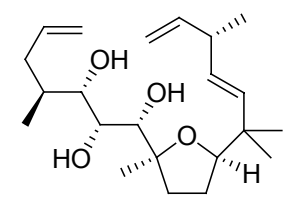

(no compound number was assigned in the manuscript)

Triol. A $25 \mathrm{~mL}$ round bottom flask was charged with the TBS-protected diol $16(141 \mathrm{mg}, 293 \mu \mathrm{mol})$ and $5 \mathrm{~mL}$ of anhydrous THF. TBAF $(215 \mathrm{mg}, 682 \mu \mathrm{mol})$ was added, and after $4 \mathrm{~h}$ the reaction was directly filtered through a short column with silica gel (hexanes/EtOAc, 10:1) to afford the desired triol, $104 \mathrm{mg}(284 \mu \mathrm{mol}, 97 \%)$, as an oil. Data are: tlc $R_{f}=0.08$ (hexanes/EtOAc, 10:1); $[\alpha]_{\mathrm{D}}{ }^{20}=$ $+14.4^{\circ}\left(c 1, \mathrm{CHCl}_{3}\right) ;{ }^{1} \mathrm{H}$ NMR $\left(400 \mathrm{MHz}, \mathrm{CDCl}_{3}\right) \delta 5.87-$ $5.73(\mathrm{~m}, 2 \mathrm{H}), 5.45(\mathrm{~d}, J=15.9 \mathrm{~Hz}, 1 \mathrm{H}), 5.38$ (dd, $J=$ $15.9,6.1 \mathrm{~Hz}, 1 \mathrm{H}), 5.08-4.90(\mathrm{~m}, 4 \mathrm{H}), 3.98$ (br s, $1 \mathrm{H})$, $3.74(\mathrm{dd}, J=8.9,6.5 \mathrm{~Hz}, 1 \mathrm{H}), 3.68-3.64(\mathrm{~m}, 1 \mathrm{H}), 3.48-$ 3.39 (m 2 H), 3.15-3.04 (m, 2 H), 2.83 (app hex, $J=6.5$ $\mathrm{Hz}, 1 \mathrm{H}), 2.42-2.34$ (m, $1 \mathrm{H}), 2.18$ (ddd, $J=12.1,9.4,4.7$ $\mathrm{Hz}, 1 \mathrm{H}), 2.03-1.94$ (m, $1 \mathrm{H}), 1.89-1.76$ (m, $2 \mathrm{H}), 1.76-1.66$ (m, $1 \mathrm{H}), 1.64-1.54(\mathrm{~m}, 1 \mathrm{H}), 1.23(\mathrm{~s}, 3 \mathrm{H}), 1.08(\mathrm{~d}, J=6.9$ $\mathrm{Hz}, 3 \mathrm{H}), 1.03(\mathrm{~s}, 3 \mathrm{H}), 0.96(\mathrm{~s}, 3 \mathrm{H}), 0.95(\mathrm{~d}, J=6.8 \mathrm{~Hz}, 3$ $\mathrm{H}) ;{ }^{13} \mathrm{C}$ NMR $\left(100 \mathrm{MHz}, \mathrm{CDCl}_{3}\right) \delta 143.1,137.8,134.4$, $132.8,116.0,112.8,86.7,86.1,77.8,76.8,69.2,40.5,38.8$, $36.3,35.4,34.9,27.0,24.6,24.4,23.6,20.0,16.1$; IR (neat) v 3457, 3076, 2962, 2929, 2871, 1727, 1638, 1458, 1384, 1272, 1073, 1054, 1024, 993, 911, 849, 792, 743, 670; HRMS (ESI) found $389.2667[\mathrm{M}+\mathrm{Na}]^{+}$, calcd 389.2668 for $\mathrm{C}_{22} \mathrm{H}_{38} \mathrm{O}_{4} \mathrm{Na}^{+}$.

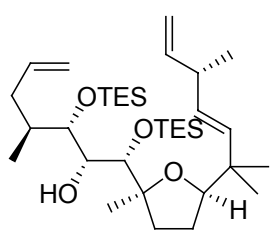

(no compound number was assigned in the manuscript) Bis-TES protected alcohol. A $25 \mathrm{~mL}$ round bottom flask was charged with the deprotected triol $(84 \mathrm{mg}, 229 \mu \mathrm{mol})$ and $12 \mathrm{~mL}$ of anhydrous DMF. Then imidazole $(95 \mathrm{mg}$, $1.40 \mathrm{mmol})$ was added, followed by TES-Cl $(120 \mu \mathrm{L}, 108$ $\mathrm{mg}, 715 \mu \mathrm{mol})$. After $20 \mathrm{~h}$ the mixture was warmed to $30^{\circ} \mathrm{C}$ and a second portion TES-Cl $(80 \mu \mathrm{L}, 72 \mathrm{mg}, 477 \mu \mathrm{mol})$ was introduced. The reaction was terminated after $6 \mathrm{~h}$ at $30^{\circ} \mathrm{C}$ by addition of saturated $\mathrm{NH}_{4} \mathrm{Cl}$ solution $(10 \mathrm{~mL})$ and hexanes $(20 \mathrm{~mL})$. Then, the aqueous layer was separated and extracted with hexanes $(3 \times 20 \mathrm{~mL})$. The combined organic solutions were dried over $\mathrm{Na}_{2} \mathrm{SO}_{4}$ and concentrated under reduced pressure. Flash chromatography (hexanes/EtOAc, 40:1) furnished the desired alcohol, 132 $\mathrm{mg}(222 \mu \mathrm{mol}, 97 \%)$, as an oil.

Data are: tlc $R_{f}=0.29$ (hexanes/EtOAc, $\left.40: 1\right) ;[\alpha]_{\mathrm{D}}{ }^{20}=$ $+9.3^{\circ}\left(\mathrm{c} 1, \mathrm{CHCl}_{3}\right) ;{ }^{1} \mathrm{H}$ NMR $\left(400 \mathrm{MHz}, \mathrm{CDCl}_{3}\right) \delta 5.83-$ $5.69(\mathrm{~m}, 2 \mathrm{H}), 5.46(\mathrm{dd}, J=15.9,1.1 \mathrm{~Hz}, 1 \mathrm{H}), 5.33(\mathrm{dd}$, $J=15.9,6.5 \mathrm{~Hz}), 5.03-4.89$ (m, $4 \mathrm{H}), 3.75(\mathrm{dd}, J=8.7,5.8$ $\mathrm{Hz}, 1 \mathrm{H}), 3.65$ (br s, $1 \mathrm{H}), 3.46$ (dd, $J=8.8,1.8 \mathrm{~Hz}, 1 \mathrm{H}$ ), 3.39 (t, $J=8.8 \mathrm{~Hz}, 1 \mathrm{H}), 2.80$ (app hex, $J=6.7 \mathrm{~Hz}, 1 \mathrm{H}$ ), $2.63(\mathrm{~d}, J=8.8 \mathrm{~Hz}, 1 \mathrm{H}), 2.19-2.10(\mathrm{~m}, 1 \mathrm{H}), 1.97-1.81$ (m, $2 \mathrm{H}), 1.71-1.56(\mathrm{~m}, 3 \mathrm{H}), 1.32$ (q, $J=10.4 \mathrm{~Hz}, 1 \mathrm{H}), 1.17$ $(\mathrm{s}, 3 \mathrm{H}), 1.07(\mathrm{~d}, J=6.9 \mathrm{~Hz}, 3 \mathrm{H}), 1.01-0.93(\mathrm{~m}, 27 \mathrm{H})$, 0.69-0.59 (m, $12 \mathrm{H}) ;{ }^{13} \mathrm{C}$ NMR (100 MHz, $\left.\mathrm{CDCl}_{3}\right) \delta 143.4$, 138.0, 135.4, 131.6, 115.5, 112.5, 86.1, 84.6, 79.1, 77.2, $72.8,40.4,39.3,34.86,34.85,34.3,26.0,24.2,24.1,20.0$, 18.4, 18.0, 7.1, 7.0; IR (neat) $v$ 3508, 3077, 2956, 2911, $2875,1731,1639,1459,1414,1378,1269,1238,1139$, 1114, 1093, 1066, 1006, 976, 911, 846, 811, 741, 673; HRMS (ESI) found $617.4381[\mathrm{M}+\mathrm{Na}]^{+}$, calcd 617.4397 for $\mathrm{C}_{34} \mathrm{H}_{66} \mathrm{Si}_{2} \mathrm{O}_{4} \mathrm{Na}^{+}$.

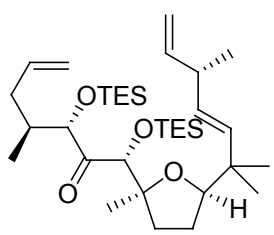

(no compound number was assigned in the manuscript) TES ketone. A $50 \mathrm{~mL}$ round bottom flask was charged with the TES alcohol $(115 \mathrm{mg}, 193 \mu \mathrm{mol})$ and $15 \mathrm{~mL}$ of dry $\mathrm{CH}_{2} \mathrm{Cl}_{2}$. The solution was then charged with DessMartin periodinane $(342 \mathrm{mg}, 806 \mu \mathrm{mol})$ and stirred for 1.5 $\mathrm{h}$. The reaction mixture was terminated by addition of saturated $\mathrm{NaHCO}_{3}$ solution $(3 \mathrm{~mL})$ and saturated $\mathrm{Na}_{2} \mathrm{~S}_{2} \mathrm{O}_{3}$ solution $(3 \mathrm{~mL})$ and vigorously stirred for 30 minutes. The aqueous layer was separated and extracted once with $\mathrm{CH}_{2} \mathrm{Cl}_{2}(5 \mathrm{~mL})$. The organic solutions were dried over $\mathrm{Na}_{2} \mathrm{SO}_{4}$ and concentrated under reduced pressure. Flash chromatography (hexanes/EtOAc, 40:1) afforded the desired ketone, $106 \mathrm{mg}(179 \mu \mathrm{mol}, 93 \%)$, as an oil.

Data are: tlc $R_{f}=0.39$ (hexanes/EtOAc, 40:1), 0.74 (hexanes/EtOAc, 10:1); $[\alpha]_{\mathrm{D}}{ }^{20}=+40.1^{\circ}\left(c 1, \mathrm{CHCl}_{3}\right) ;{ }^{1} \mathrm{H}$ NMR $\left(400 \mathrm{NHz}, \mathrm{CDCl}_{3}\right) \delta 5.82-5.64(\mathrm{~m}, 2 \mathrm{H}), 5.44$ (dd, $J$ 
$=15.9 \mathrm{~Hz}, 0.8 \mathrm{~Hz}, 1 \mathrm{H}), 5.32(\mathrm{dd}, J=15.9,6.7 \mathrm{~Hz}, 1 \mathrm{H})$, 5.01-4.89 (m, $4 \mathrm{H}), 4.66$ (d, $J=2.6 \mathrm{~Hz}, 1 \mathrm{H}), 4.20$ (br s, 1 H), 3.68 (dd, $J=7.8,6.3 \mathrm{~Hz}, 1 \mathrm{H}$ ), 2.80 (app hex, $J=6.7$ $\mathrm{Hz}, 1 \mathrm{H}), 2.13-2.03$ (m, $1 \mathrm{H}), 2.02-1.94$ (m, $1 \mathrm{H}), 1.93-1.78$ (m, $3 \mathrm{H}), 1.64-1.53(\mathrm{~m}, 1 \mathrm{H}), 1.49-1.36(\mathrm{~m}, 1 \mathrm{H}), 1.09(\mathrm{~s}, 3$ H), 1.07 (d, $J=6.9 \mathrm{~Hz}, 3 \mathrm{H}), 1.03-0.92(\mathrm{~m}, 27 \mathrm{H}), 0.80-$ $0.71(\mathrm{~m}, 6 \mathrm{H}), 0.64-0.55(\mathrm{~m}, 6 \mathrm{H}) ;{ }^{13} \mathrm{C} \mathrm{NMR}(100 \mathrm{MHz}$, $\left.\mathrm{CDCl}_{3}\right) \delta 209.2,143.3,137.4,135.5,131.6,115.9,112.5$, $84.8,84.1,81.5,79.4,40.4,39.1,36.3,34.6,34.5,26.2$, $24.4,24.1,20.0,18.1,17.0,7.0,6.8,5.0,4.9$; IR (neat) $v$ 3078, 2957, 2911, 2876, 1733, 1640, 1459, 1415, 1372, 1330, 1239, 1170, 1095, 1006, 976, 912, 836, 742, 683; HRMS (ESI) found 615.4268 [M + Na $]^{+}$, calcd 615.4241 for $\mathrm{C}_{34} \mathrm{H}_{64} \mathrm{Si}_{2} \mathrm{O}_{4} \mathrm{Na}^{+}$.

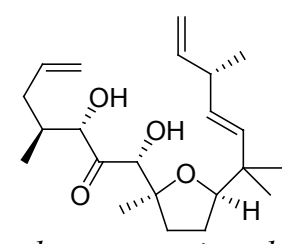

(no compound number was assigned in the manuscript) Hydroxy ketone. A $10 \mathrm{~mL}$ round bottom flask was charged with the TES ketone $(205 \mathrm{mg}, 346 \mu \mathrm{mol})$ and $3 \mathrm{~mL}$ of anhydrous THF. After cooling to $0^{\circ} \mathrm{C}$ TBAF $(298$ $\mathrm{mg}, 944 \mu \mathrm{mol})$ in $3 \mathrm{~mL}$ of dry THF was added, and after 30 minutes the reaction was stopped by direct filtration of the mixture (silica gel; hexanes/EtOAc, 10:1) to furnish the desired hydroxyl ketone, $125 \mathrm{mg}$ (343 $\mu \mathrm{mol}, 99 \%)$, as an oil.

Data are: tlc $R_{f}=0.32$ (hexanes/EtOAc, $\left.10: 1\right) ;[\alpha]_{\mathrm{D}}{ }^{20}=$ $64.1^{\circ}$ (c 1, $\left.\mathrm{CHCl}_{3}\right) ;{ }^{1} \mathrm{H}$ NMR $\left(400 \mathrm{MHz}, \mathrm{CDCl}_{3}\right) \delta 5.76$ (ddd, $J=17.2,10.4,6.7 \mathrm{~Hz}, 1 \mathrm{H}), 5.74-5.63(\mathrm{~m}, 1 \mathrm{H}), 5.37$ $(\mathrm{dd}, J=15.8 \mathrm{~Hz}, 5.6 \mathrm{~Hz}, 1 \mathrm{H}), 5.31(\mathrm{~d}, J=15.8 \mathrm{~Hz}, 1 \mathrm{H})$, 5.06-4.91 (m, $4 \mathrm{H}), 4.49$ (d, $J=6.2 \mathrm{~Hz}, 1 \mathrm{H}), 4.31$ (br s, 1 H), 4.15 (br s, $1 \mathrm{H}), 3.81$ (t, $J=7.0 \mathrm{~Hz}, 1 \mathrm{H}), 3.18$ (d, $J=$ $6.2 \mathrm{~Hz}, 1 \mathrm{H}), 2.81($ app hex, $J=6.6 \mathrm{~Hz}, 1 \mathrm{H}), 2.22-2.12(\mathrm{~m}$, $1 \mathrm{H}), 2.10-2.02(\mathrm{~m}, 1 \mathrm{H}), 2.02-1.92(\mathrm{~m}, 1 \mathrm{H}), 1.89-1.77(\mathrm{~m}$, $2 \mathrm{H}), 1.68-1.52(\mathrm{~m}, 2 \mathrm{H}), 1.30(\mathrm{~s}, 3 \mathrm{H}), 1.08(\mathrm{~d}, J=6.9 \mathrm{~Hz}$, $3 \mathrm{H}), 1.02$ (d, $J=6.9 \mathrm{~Hz}, 3 \mathrm{H}), 0.98$ (s, $3 \mathrm{H}), 0.92(\mathrm{~s}, 3 \mathrm{H})$, ${ }^{13} \mathrm{C}$ NMR $\left(100 \mathrm{MHz}, \mathrm{CDCl}_{3}\right) \delta 216.0,142.9,136.9,134.2$, 132.9, 116.4, 112.8, 86.4, 85.6, 79.8, 78.4, 40.4, 38.8, 37.2, $35.3,32.0,25.3,24.8,23.6,23.3,19.9,16.5$; IR (neat) $v$ 3459, 3078, 2969, 2932, 2873, 1709, 1640, 1456, 1374, $1265,1123,1071,1022,995,913,851$; HRMS (ESI) found $387.2521[\mathrm{M}+\mathrm{Na}]^{+}$, calcd 387.2511 for $\mathrm{C}_{22} \mathrm{H}_{36} \mathrm{O}_{4} \mathrm{Na}^{+}$.

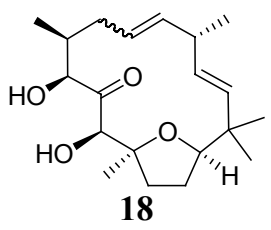

Trans olefin 18. A $500 \mathrm{~mL}$ round bottom flask with a reflux condenser was charged with the deprotected hydroxyl ketone $(125 \mathrm{mg}, 343 \mu \mathrm{mol})$ and $200 \mathrm{~mL}$ of anhydrous $\mathrm{CH}_{2} \mathrm{Cl}_{2}$. Then Grubbs II catalyst $(27.8 \mathrm{mg}, 32.7$ $\mu \mathrm{mol})$ was added and the reaction was refluxed over $1.5 \mathrm{~h}$ before cooling to room temperature. The solution was saturated with air and silica gel (400 mg) was added. After concentration the resulting solid was purified by flash column chromatography (hexanes/EtOAc, 10:1) to afford the macrocycle 18, $98 \mathrm{mg}(290 \mu \mathrm{mol}, 85 \%)$, as an oil $(E: Z$ $\approx 5: 1$ ).

Data are: tlc $R_{f}=0.13$ (hexanes/EtOAc, $10: 1$ ); $[\alpha]_{\mathrm{D}}{ }^{20}=$ $+48.2^{\circ}$ (c 1, $\left.\mathrm{CHCl}_{3}\right) ;{ }^{1} \mathrm{H} \mathrm{NMR}\left(400 \mathrm{MHz}, \mathrm{CDCl}_{3}\right) \delta 5.40$ (dd, $J=15.5,8.8 \mathrm{~Hz}, 1 \mathrm{H}), 5.33$ (d, $J=15.4 \mathrm{~Hz}, 1 \mathrm{H}), 5.27$ $(\mathrm{dd}, J=15.4 \mathrm{~Hz}, 7.9 \mathrm{~Hz}, 1 \mathrm{H}), 5.24-5.16(\mathrm{~m}, 1 \mathrm{H}), 4.57$ (br s, $1 \mathrm{H}), 4.35$ (br s, $1 \mathrm{H}), 3.81(\mathrm{dd}, J=8.0,3.0 \mathrm{~Hz}, 1 \mathrm{H})$, 3.54 (br s, $1 \mathrm{H}), 2.80-2.67(\mathrm{~m}, 2 \mathrm{H}), 2.57-2.47(\mathrm{~m}, 1 \mathrm{H})$, 2.09-1.97 (m, 2 H), 1.95-1.74 (m, 4 H), 1.17 (d, J=7.0 Hz, $3 \mathrm{H}), 1.09$ (s, $3 \mathrm{H}), 1.05$ (s, $3 \mathrm{H}), 1.03$ (d, $J=6.7 \mathrm{~Hz}, 3 \mathrm{H})$, $0.88(\mathrm{~s}, 3 \mathrm{H}) ;{ }^{13} \mathrm{C} \mathrm{NMR}\left(100 \mathrm{MHz}, \mathrm{CDCl}_{3}\right) \delta 212.4,137.2$, 133.8, 132.7, 127.8, 88.2, 85.4, 81.8, 77.9, 42.0, 40.3, 39.6, $34.6,32.3,26.7,25.7,24.2,21.3,21.0,20.4$; IR (neat) $v$ $3477,2961,2930,2871,1707,1454,1374,1327,1239$, 1156, 1121, 1074, 1059, 1024, 982, 910, 883, 829, 769, 726, 666; HRMS (ESI) found 359.2202 [M + Na] $]^{+}$, calcd 359.2198 for $\mathrm{C}_{20} \mathrm{H}_{32} \mathrm{O}_{4} \mathrm{Na}^{+}$.

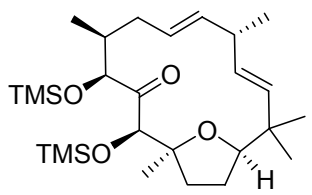

(no compound number was assigned in the manuscript) TMS olefin. A $25 \mathrm{~mL}$ round bottom flask was charged with olefin $18(28 \mathrm{mg}, 83.2 \mu \mathrm{mol})$ and $10 \mathrm{~mL}$ of anhydrous DMF. The solution was cooled to $0^{\circ} \mathrm{C}$ before $\mathrm{NEt}_{3}(80 \mu \mathrm{L}$, $58.4 \mathrm{mg}, 577 \mu \mathrm{mol})$ and TMS-Cl $(50 \mu \mathrm{L}, 43 \mathrm{mg}, 395$ $\mu \mathrm{mol})$ were added consecutively. After $1 \mathrm{~h}$ saturated $\mathrm{NH}_{4} \mathrm{Cl}$ solution $(5 \mathrm{~mL})$ was added, followed by $\mathrm{H}_{2} \mathrm{O}(15$ $\mathrm{mL})$ and hexanes/EtOAc $(10: 1 ; 30 \mathrm{~mL})$. The organic layer was washed twice with $\mathrm{H}_{2} \mathrm{O}(20 \mathrm{~mL})$, and the combined aqueous phases were reextracted once with hexanes/EtOAc $(10: 1 ; 20 \mathrm{~mL})$. Then, the organic solutions were combined, dried over $\mathrm{Na}_{2} \mathrm{SO}_{4}$ and concentrated under reduced pressure. A short filtration over silica gel (hexanes/EtOAc, 10:1) afforded the TMS-protected diol, $39.8 \mathrm{mg}(82.8$ $\mu \mathrm{mol},>99 \%$ ), as an oil.

Data are: tlc $R_{f}=0.67$ (hexanes/EtOAc, $10: 1$ ); $[\alpha]_{\mathrm{D}}{ }^{20}=-$ $23.4^{\circ}\left(c \quad 0.5, \mathrm{CHCl}_{3}\right)$; ${ }^{1} \mathrm{H}$ NMR $\left(400 \mathrm{MHz}, \mathrm{CDCl}_{3}\right) \delta 5.54-$ $5.24(\mathrm{~m}, 4 \mathrm{H}), 4.75-4.63(\mathrm{~m}, 1 \mathrm{H}), 4.50$ (br d, $J=1.8 \mathrm{~Hz}, 1$ H), 3.62 (br t, $J=6.0 \mathrm{~Hz}, 1 \mathrm{H}), 2.74$ (app hex, $J=6.5 \mathrm{~Hz}, 1$ H), 2.52-2.33 (m, 1 H), 2.13-1.98 (m, $1 \mathrm{H}), 1.96-1.66$ (m, 4 $\mathrm{H}), 1.64-1.46(\mathrm{~m}, 1 \mathrm{H}), 0.87(\mathrm{~s}, 3 \mathrm{H}), 1.08-0.98(\mathrm{~m}, 12 \mathrm{H})$, $0.18(\mathrm{~s}, 9 \mathrm{H}), 0.12(\mathrm{~s}, 9 \mathrm{H}),{ }^{13} \mathrm{C} \mathrm{NMR}\left(100 \mathrm{MHz}, \mathrm{CDCl}_{3}\right) \delta$ $136.7,134.6,132.3,127.3,87.2,86.4,85.6,78.3,40.2$, $39.0,34.0,32.1,26.3,26.1,24.9,24.6,21.9,20.1,15.5$, $0.7,0.1, \mathrm{C}=\mathrm{O}$ was not observed, the NMR spectra display a strong line broadening due to a slow conformational equilibrium; IR (neat) v 2960, 2871, 1727, 1453, 1371, 1305, 1251, 1099, 1051, 1027, 999, 980, 880, 842, 753; HRMS (ESI) found 503.2986 [M + Na] ${ }^{+}$, calcd 503.2989 for $\mathrm{C}_{26} \mathrm{H}_{48} \mathrm{O}_{4} \mathrm{Si}_{2} \mathrm{Na}^{+}$. 


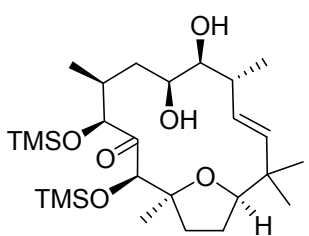

(no compound number was assigned in the manuscript) TMS diol. A $10 \mathrm{~mL}$ round bottom flask was charged with the TMS ether of $\mathbf{1 8}(62 \mathrm{mg}, 129 \mu \mathrm{mol}), t \mathrm{BuOH}(4 \mathrm{~mL})$ and $\mathrm{H}_{2} \mathrm{O}(4 \mathrm{~mL})$. Then $\alpha$-DHQ-CLB $(21.8 \mathrm{mg}, 46.9 \mu \mathrm{mol})$, $\mathrm{K}_{2} \mathrm{CO}_{3}(55.5 \mathrm{mg}, 402 \mu \mathrm{mol}), \mathrm{K}_{3} \mathrm{Fe}(\mathrm{CN})_{6}(129 \mathrm{mg}, 392$ $\mu \mathrm{mol})$ and MSA (37 mg, $389 \mu \mathrm{mol})$ were added consecutively. When the mixture became a clear the solution was cooled to $0^{\circ} \mathrm{C}$ and an $\mathrm{OsO}_{4}$ solution $(2.5 \mathrm{wt}-\%$ in $t \mathrm{BuOH}, 162 \mu \mathrm{L}, 131 \mu \mathrm{mg}, 12.9 \mu \mathrm{mol})$ was introduced dropwise. After $2.5 \mathrm{~h}$ the reaction was terminated by addition of an halfsaturated aqueous $\mathrm{Na}_{2} \mathrm{~S}_{2} \mathrm{O}_{3}$ solution. The mixture was extracted with $\mathrm{CH}_{2} \mathrm{Cl}_{2}(3 \times 5 \mathrm{~mL})$, and the combined organic phases were dried over $\mathrm{Na}_{2} \mathrm{SO}_{4}$ and concentrated under reduced pressure. Flash chromatography (hexanes/EtOAc, 5:1) afforded the title compound, $55 \mathrm{mg}(107 \mu \mathrm{mol}, 83 \%)$, as an oil.

Data are: tlc $R_{f}=0.17$ (hexanes/EtOAc, $5: 1$ ); $[\alpha]_{\mathrm{D}}{ }^{20}=$ $+4.8^{\circ},[\alpha]_{365}{ }^{20}=-12.0^{\circ}\left(c 1, \mathrm{CHCl}_{3}\right) ;{ }^{1} \mathrm{H}$ NMR $(400 \mathrm{MHz}$, $\left.\mathrm{CDCl}_{3}\right) \delta 5.49(\mathrm{~d}, J=15.8 \mathrm{~Hz}, 1 \mathrm{H}), 5.12(\mathrm{dd}, J=15.8,9.2$ Hz, $1 \mathrm{H}$ ), 4.65 (br s, $1 \mathrm{H}), 4.29$ (br s, $1 \mathrm{H}), 3.67$ (br s, $1 \mathrm{H}$ ), $3.56(\mathrm{dd}, J=9.6,5.6 \mathrm{~Hz}, 1 \mathrm{H}), 3.15$ (br t, $J=9.6 \mathrm{~Hz}, 1 \mathrm{H})$, 2.40-2.20 (m, $2 \mathrm{H}), 2.00-1.88$ (m, $2 \mathrm{H}), 1.88-1.62$ (m, $4 \mathrm{H})$, 1.51-1.41 (m, $1 \mathrm{H}), 1.18(\mathrm{~s}, 3 \mathrm{H}), 1.11(\mathrm{~s}, 3 \mathrm{H}), 1.08(\mathrm{~d}, J=$ $6.7 \mathrm{~Hz}, 3 \mathrm{H}), 0.98$ (d, $J=6.5 \mathrm{~Hz}, 3 \mathrm{H}), 0.88$ (s, $3 \mathrm{H}), 0.16$ $(\mathrm{s}, 9 \mathrm{H}), 0.13(\mathrm{~s}, 9 \mathrm{H}),{ }^{13} \mathrm{C} \mathrm{NMR}\left(100 \mathrm{MHz}, \mathrm{CDCl}_{3}\right) \delta$ 136.3, 131.6, 86.3, 84.1, 77.6, 77.2, 74.6, 68.7, 40.6, 38.1, $35.6,34.0,31.2,27.5,26.0,25.5,25.4,18.2,16.6,0.4,0.3$, $\mathrm{C}=\mathrm{O}$ signal was not detected because the NMR spectra display a strong line broadening for which we make a slow conformational equilibrium responsible; IR (neat) v 3424, 2960, 2872, 1726, 1453, 1372, 1251, 1097, 1049, 997, 925, 878, 842, 754 ; HRMS (ESI) found $537.3060[\mathrm{M}+\mathrm{Na}]^{+}$, calcd 537.3044 for $\mathrm{C}_{26} \mathrm{H}_{50} \mathrm{O}_{6} \mathrm{Si}_{2} \mathrm{Na}^{+}$.

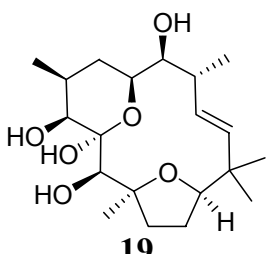

Triol 19. A $5 \mathrm{~mL}$ round bottom flask was charged with the protected dihydroxylated product $(17.7 \mathrm{mg}, 34.4 \mu \mathrm{mol})$ and $1 \mathrm{~mL}$ of dry THF. The solution was cooled to $0^{\circ} \mathrm{C}$ before a solution of TBAF $(13.2 \mathrm{mg}, 41.8 \mu \mathrm{mol})$ in $0.5 \mathrm{~mL}$ of dry THF was added. After 10 minutes the complete mixture was directly filtered over a short plug of silica gel (hexanes/EtOAc, 1:1) and the resulting solution was concentrated under reduced pressure. The colourless oil was dried in vacuum to afford the title compound, $12.7 \mathrm{mg}$ (34.3 $\mu \mathrm{mol},>99 \%)$.

Data are: tlc $R_{f}=0.17$ (hexanes/EtOAc, 1:1), 0.27 (EtOAc); $[\alpha]_{\mathrm{D}}{ }^{20}=+30.6^{\circ}\left(c\right.$ 0.5, MeOH); ${ }^{1} \mathrm{H}$ NMR $\left(400 \mathrm{MHz}, \mathrm{d}_{4^{-}}\right.$ $\mathrm{MeOH}) \delta 5.49(\mathrm{~d}, J=15.3 \mathrm{~Hz}, 1 \mathrm{H}), 5.36(\mathrm{dd}, J=15.3,9.3$
$\mathrm{Hz}, 1 \mathrm{H}), 4.12$ (dt, $J=12.3,2.3 \mathrm{~Hz}, 1 \mathrm{H}), 3.86$ (dd, $J=7.8$, $6.2 \mathrm{~Hz}, 1 \mathrm{H}), 3.72$ (br s, $1 \mathrm{H}), 3.34$ (d, $J=2.4 \mathrm{~Hz}, 1 \mathrm{H})$, 3.27 (dd, $J=6.3,2.3 \mathrm{~Hz}, 1 \mathrm{H}), 2.35-2.15$ (m, $3 \mathrm{H}), 2.08-$ $1.92(\mathrm{~m}, 2 \mathrm{H}), 1.74(\mathrm{q}, J=12.3 \mathrm{~Hz}, 1 \mathrm{H}), 1.61$ (ddd, $J=$ 12.2, 7.2, $5.1 \mathrm{~Hz}, 1 \mathrm{H}), 1.31(\mathrm{~s}, 3 \mathrm{H}), 1.13-1.04(\mathrm{~m}, 1 \mathrm{H})$, $1.09(\mathrm{~d}, J=7.0 \mathrm{~Hz}, 3 \mathrm{H}), 1.07(\mathrm{~s}, 3 \mathrm{H}), 0.94(\mathrm{~d}, J=7.0 \mathrm{~Hz}$, $3 \mathrm{H}), 0.92(\mathrm{~s}, 3 \mathrm{H}),{ }^{1} \mathrm{H}$ NMR $\left(500 \mathrm{MHz}, \mathrm{CDCl}_{3}\right) \delta 5.45(\mathrm{~d}$, $J=15.4 \mathrm{~Hz}, 1 \mathrm{H}), 5.31(\mathrm{dd}, J=15.4,9.2 \mathrm{~Hz}, 1 \mathrm{H}), 4.56(\mathrm{br}$ s, $1 \mathrm{H}), 4.12(\mathrm{dt}, J=12.1,2.1 \mathrm{~Hz}, 1 \mathrm{H}), 3.88$ (bs, $1 \mathrm{H}), 3.81$ (t, $J=7.2 \mathrm{~Hz}, 1 \mathrm{H}), 3.67$ (br s, $1 \mathrm{H}), 3.40$ (bs, $1 \mathrm{H}), 3.27$ (bd, $J=4.6 \mathrm{~Hz}, 1 \mathrm{H}), 2.39-2.26(\mathrm{~m}, 3 \mathrm{H}), 1.98-1.84(\mathrm{~m}, 2$ $\mathrm{H}), 1.74$ (ps q, $J=12.9 \mathrm{~Hz}, 1 \mathrm{H}), 1.66-1.54(\mathrm{~m}, 2 \mathrm{H}), 1.30$ $(\mathrm{s}, 3 \mathrm{H}), 1.22-1.14(\mathrm{~m}, 1 \mathrm{H}), 1.11(\mathrm{~d}, J=7.0 \mathrm{~Hz}, 3 \mathrm{H}), 1.08$ $(\mathrm{s}, 3 \mathrm{H}), 0.97(\mathrm{~d}, J=7.0 \mathrm{~Hz}, 3 \mathrm{H}), 0.91(\mathrm{~s}, 3 \mathrm{H}),{ }^{13} \mathrm{C} \mathrm{NMR}$ $\left(100 \mathrm{MHz}, \mathrm{d}_{4}-\mathrm{MeOH}\right) \delta 136.0,131.6,98.6,89.46,89.44$, $81.4,79.5,76.1,70.5,46.7,39.8,35.6,31.0,30.2,27.9$, 26.8, 26.6, 24.7, 21.5, 17.8; IR (neat) v 3348, 2963, 2927, 2870, 1719, 1665, 1611, 1547, 1455, 1369, 1286, 1225, 1120, 1098, 1059, 1024, 991, 870, 778, 702; HRMS (ESI) found $393.2260[\mathrm{M}+\mathrm{Na}]^{+}$, calcd 393.2253 for $\mathrm{C}_{20} \mathrm{H}_{34} \mathrm{O}_{6} \mathrm{Na}^{+}$.

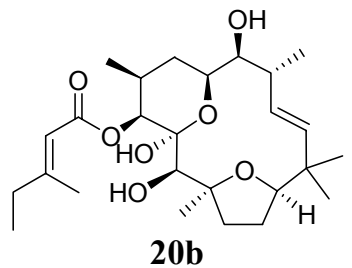

20b. A $5 \mathrm{~mL}$ round bottom flask was charged with 19 (5.5 $\mathrm{mg}, 14.8 \mu \mathrm{mol})$, the side chain $(25.2 \mathrm{mg}, 221 \mu \mathrm{mol})$ and $1.2 \mathrm{~mL}$ of dry $\mathrm{CH}_{2} \mathrm{Cl}_{2}$. DIC $(33 \mu \mathrm{L}, 26.9 \mathrm{mg}, 213 \mu \mathrm{mol})$ was added via syringe, followed by DMAP $(2.1 \mathrm{mg}, 17.2$ $\mu \mathrm{mol})$ after $1 \mathrm{~h}$. The reaction mixture was allowed to stir for $28 \mathrm{~h}$ at room temperature and then directly filtered over silica gel (hexanes/EtOAc, 1:1). The resulting filtrate was reduced and purified by flash column chromatography (hexanes/EtOAc, 10:1 $\rightarrow$ 5:1 $\rightarrow$ 2.5:1) to afford 20b and 20c, $4.8 \mathrm{mg}(70 \%)$, as an oil and inseparable, along with 20a, $1.5 \mathrm{mg}(22 \%)$, as an oil. 20a was fully characterized, but tends to spontaneously rearrange to the desired $\mathbf{2 0 b}$, thereby furnishing 20b as pure material. 20c was obtained as sole product under Yamaguchi conditions, so that the following spectroscopical data for 20a-c were all recorded for pure samples.

Data are (20b): tlc $R_{f}=0.63$ (hexanes/EtOAc, $\left.1: 1\right) ;[\alpha]_{\mathrm{D}}{ }^{20}=$ $+16.3^{\circ}\left(\mathrm{c} 0.08, \mathrm{CHCl}_{3}\right) ;{ }^{1} \mathrm{H}$ NMR $\left(400 \mathrm{MHz}, \mathrm{CDCl}_{3}\right) \delta 6.22$ $(\mathrm{s}, 1 \mathrm{H}), 5.70(\mathrm{q}, J=1.2 \mathrm{~Hz}, 1 \mathrm{H}), 5.50(\mathrm{~d}, J=15.3 \mathrm{~Hz}, 1$ H), $5.28(\mathrm{dd}, J=15.3,9.3 \mathrm{~Hz}, 1 \mathrm{H}), 4.92(\mathrm{~d}, J=2.5 \mathrm{~Hz}, 1$ H), $4.20(\mathrm{dt}, J=12.0,2.3 \mathrm{~Hz}, 1 \mathrm{H}), 3.70(\mathrm{dd}, J=9.5,5.9$ $\mathrm{Hz}, 1 \mathrm{H}), 3.46(\mathrm{~d}, J=6.4 \mathrm{~Hz}, 1 \mathrm{H}), 3.38$ (ddd, $J=8.5,6.3$, $2.3 \mathrm{~Hz}, 1 \mathrm{H}), 3.07$ (d, $J=6.4 \mathrm{~Hz}, 1 \mathrm{H}), 2.48-2.27$ (m, $3 \mathrm{H})$, 2.24-2.11 (m, 5 H), 1.94-1.83 (m, $1 \mathrm{H}), 1.82-1.72(\mathrm{~m}, 2 \mathrm{H})$, 1.49 (ddd, $J=12.4,9.9,7.3 \mathrm{~Hz}, 1 \mathrm{H}), 1.32$ (s, $3 \mathrm{H}), 1.20-$ $1.16(\mathrm{~m}, 1 \mathrm{H}), 1.12(\mathrm{~d}, J=7.0 \mathrm{~Hz}, 3 \mathrm{H}), 1.10(\mathrm{~s}, 3 \mathrm{H}), 1.07$ (t, $J=7.5 \mathrm{~Hz}, 3 \mathrm{H}), 0.90(\mathrm{~s}, 3 \mathrm{H}), 0.84(\mathrm{~d}, J=6.9 \mathrm{~Hz}, 3 \mathrm{H})$; ${ }^{13} \mathrm{C} \mathrm{NMR}\left(100 \mathrm{MHz}, \mathrm{CDCl}_{3}\right) \delta 166.6,135.0,130.4,114.1$, $96.8,88.0,87.9,78.3,78.1,73.5,68.4,45.5,38.4,36.2$, $33.9,30.0,29.1,28.0,27.3,26.0,25.2,20.9,19.0,17.3$, 11.9, C-3' was not detected; IR (neat) v 3354, 2969, 2925, 
$2855,1741,1670,1653,1456,1374,1261,1227,1216$, 1144, 1074, 1023, 798, 696 ; HRMS (ESI) found 489.2840 $[\mathrm{M}+\mathrm{Na}]^{+}$, calcd 489.2828 for $\mathrm{C}_{26} \mathrm{H}_{42} \mathrm{O}_{7} \mathrm{Na}^{+}$.

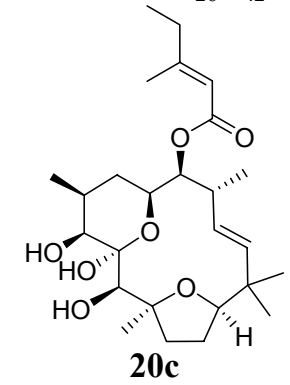

Data are (20c): tlc $R_{f}=0.59$ (hexanes/EtOAc, $\left.1: 1\right) ;[\alpha]_{\mathrm{D}}{ }^{20}=$ $+25.2^{\circ}\left(c 0.25, \mathrm{CHCl}_{3}\right) ;{ }^{1} \mathrm{H}$ NMR $\left(400 \mathrm{MHz}, \mathrm{CDCl}_{3}\right) \delta 6.14$ $(\mathrm{s}, 1 \mathrm{H}), 5.72(\mathrm{q}, J=1.2 \mathrm{~Hz}, 1 \mathrm{H}), 5.54(\mathrm{~d}, J=15.3 \mathrm{~Hz}, 1$ H), 5.33 (dd, $J=15.3,9.4 \mathrm{~Hz}, 1 \mathrm{H}$ ), 4.92 (dd, $J=7.7,3.1$ $\mathrm{Hz}, 1 \mathrm{H}), 4.31$ (dt, $J=11.9,3.1 \mathrm{~Hz}, 1 \mathrm{H}), 3.75(\mathrm{dd}, J=9.0$, $6.0 \mathrm{~Hz}, 1 \mathrm{H}), 3.68-3.63(\mathrm{~m}, 1 \mathrm{H}), 3.32$ (br s, $1 \mathrm{H}), 2.51-2.39$ (m, $2 \mathrm{H}), 2.25-2.13(\mathrm{~m}, 4 \mathrm{H}), 2.18(\mathrm{~d}, J=1.2 \mathrm{~Hz}, 3 \mathrm{H})$, 1.97-1.73 (m, $4 \mathrm{H}), 1.34$ (s, $3 \mathrm{H}), 1.30-1.21(\mathrm{~m}, 1 \mathrm{H}), 1.12$ $(\mathrm{s}, 3 \mathrm{H}), 1.10(\mathrm{t}, J=7.5 \mathrm{~Hz}, 3 \mathrm{H}), 1.01(\mathrm{~d}, J=6.9 \mathrm{~Hz}, 3 \mathrm{H})$, $0.93(\mathrm{~d}, J=6.9 \mathrm{~Hz}, 3 \mathrm{H}), 0.90(\mathrm{~s}, 3 \mathrm{H})$, the ${ }^{1} \mathrm{H}$ NMR spectrum shows slightly broadened signals for which we make conformational equilibrium responsible; ${ }^{13} \mathrm{C}$ NMR $\left(100 \mathrm{MHz}, \mathrm{CDCl}_{3}\right) \delta 166.9,162.5,135.6,129.7,114.3$, 97.1, 88.1, 87.9, 79.1, 77.2, 77.9, 68.4, 42.3, 38.5, 36.0, $33.9,29.3,28.4,27.9,27.3,25.8,24.8,20.6,18.9,17.3$, 11.9; IR (neat) $\vee$ 3500, 3351, 2963, 2926, 2872, 1713, $1648,1456,1375,1261,1217,1144,1099,1070,1034$, 995, 866, 761; HRMS (ESI) found 489.2830 [M + Na] $]^{+}$, calcd 489.2828 for $\mathrm{C}_{26} \mathrm{H}_{42} \mathrm{O}_{7} \mathrm{Na}^{+}$.

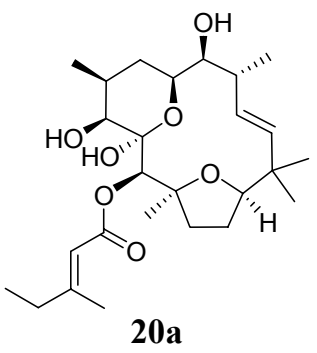

Data are (20a): tlc $R_{f}=0.32$ (hexanes/EtOAc, $\left.1: 1\right) ;[\alpha]^{20}{ }_{\mathrm{D}}=$ $+4.5^{\circ},[\alpha]^{20}{ }_{365 \mathrm{~nm}}=+30.7^{\circ}\left(c 0.31, \mathrm{CHCl}_{3}\right) ;{ }^{1} \mathrm{H}$ NMR $(400$ $\left.\mathrm{MHz}, \mathrm{CDCl}_{3}\right) \delta 5.83(\mathrm{~s}, 1 \mathrm{H}), 5.64(\mathrm{q}, J=1.3 \mathrm{~Hz}, 1 \mathrm{H})$, $5.46(\mathrm{~d}, J=15.8 \mathrm{~Hz}, 1 \mathrm{H}), 5.33(\mathrm{dd}, J=15.8,8.2 \mathrm{~Hz}, 1 \mathrm{H})$, $5.18(\mathrm{~s}, 1 \mathrm{H}), 4.09(\mathrm{dd}, J=12.4,2.2 \mathrm{~Hz}, 1 \mathrm{H}), 3.78(\mathrm{t}, J=$ $7.3 \mathrm{~Hz}, 1 \mathrm{H}), 3.41$ (dd, $J=8.3,2.2 \mathrm{~Hz}, 1 \mathrm{H}), 3.29$ (br s, 1 H), 3.11-2.97 (br s, $1 \mathrm{H}), 2.45-2.32(\mathrm{~m}, 1 \mathrm{H}), 2.27-2.14(\mathrm{~m}$, $6 \mathrm{H}), 2.11-1.99(\mathrm{~m}, 1 \mathrm{H}), 1.94-1.76(\mathrm{~m}, 3 \mathrm{H}), 1.71-1.63(\mathrm{~m}$, $2 \mathrm{H}), 1.25(\mathrm{~s}, 3 \mathrm{H}), 1.11(\mathrm{~s}, 3 \mathrm{H}), 1.08(\mathrm{t}, J=7.5 \mathrm{~Hz}, 3 \mathrm{H})$, $1.03(\mathrm{~d}, J=7.2 \mathrm{~Hz}, 3 \mathrm{H}), 0.95(\mathrm{~s}, 3 \mathrm{H}), 0.92(\mathrm{~d}, J=6.9 \mathrm{~Hz}$, $3 \mathrm{H}) ;{ }^{13} \mathrm{C}$ NMR $\left(100 \mathrm{MHz}, \mathrm{CDCl}_{3}\right) \delta 166.0,163.9,133.7$, 131.6, 113.9, 98.0, 87.8, 86.2, 78.1, 77.2, 73.8, 66.9, 43.3, $38.4,35.3,33.9,30.7,29.9,27.2,26.8,25.78,25.75,19.4$, 19.0, 17.3, 11.9; IR (neat) v 3366, 2962, 2924, 2872, 2854, 1716, 1649, 1458, 1377, 1274, 1218, 1142, 1097, 1072, 1029, 994, 865 ; HRMS (ESI) found $489.2837[\mathrm{M}+\mathrm{Na}]^{+}$, calcd 489.2828 for $\mathrm{C}_{26} \mathrm{H}_{42} \mathrm{O}_{7} \mathrm{Na}^{+}$.

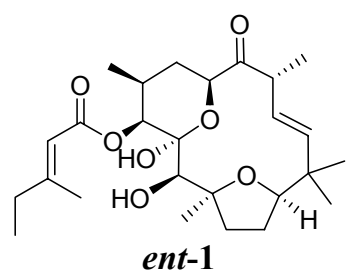

ent-1. A $5 \mathrm{~mL}$ round bottom flask was charged with 20b/20c (3.2 mg, $6.86 \mu \mathrm{mol}$ ), powdered $4 \AA$ molecular sieve $(12 \mathrm{mg})$, NMO $(10 \mathrm{mg}, 85.5 \mu \mathrm{mol})$ and $1 \mathrm{~mL}$ of dry $\mathrm{CH}_{2} \mathrm{Cl}_{2}$. TPAP $(1 \mathrm{mg}, 2.85 \mu \mathrm{mol})$ was added and the reaction was stirred at room temperature for $1 \mathrm{~h}$. Then the mixture was cautiously reduced and directly purified by flash column chromatography (hexanes/EtOAc, 10:1) to afford ent-1, $1.4 \mathrm{mg}(3.0 \mu \mathrm{mol}, 44 \%)$, as an oil, and the product of 20c oxidation, $1.3 \mathrm{mg}(2.80 \mu \mathrm{mol}, 41 \%)$, as an oil.

Data are (ent-1): tlc $R_{f}=0.66$ (hexanes/EtOAc, 2.5:1); $[\alpha]^{20}{ }_{\mathrm{D}}=-119.0^{\circ}\left(c 0.056, \mathrm{CHCl}_{3}\right) ;{ }^{1} \mathrm{H}$ NMR $(400 \mathrm{MHz}$, $\left.\mathrm{CDCl}_{3}\right) \delta 5.86(\mathrm{~d}, J=15.3 \mathrm{~Hz}, 1 \mathrm{H}), 5.70(\mathrm{q}, J=1.3 \mathrm{~Hz}, 1$ H), $5.66(\mathrm{~s}, 1 \mathrm{H}), 5.24(\mathrm{dd}, J=15.3,9.7 \mathrm{~Hz}, 1 \mathrm{H}), 4.90(\mathrm{~d}, J$ $=2.6 \mathrm{~Hz}, 1 \mathrm{H}), 4.63(\mathrm{dd}, J=11.8,2.9 \mathrm{~Hz}, 1 \mathrm{H}), 3.77(\mathrm{dd}, J$ $=11.3,5.1 \mathrm{~Hz}, 1 \mathrm{H}), 3.43(\mathrm{~d}, J=6.4 \mathrm{~Hz}, 1 \mathrm{H}), 3.34(\mathrm{dq}, J=$ 9.7, $6.8 \mathrm{~Hz}, 1 \mathrm{H}), 3.11(\mathrm{~d}, J=6.4 \mathrm{~Hz}, 1 \mathrm{H}), 2.44(\mathrm{dd}, J=$ 12.7, 7.2 Hz, $1 \mathrm{H}), 2.38-2.29(\mathrm{~m}, 1 \mathrm{H}), 2.21(\mathrm{q}, J=7.6 \mathrm{~Hz}$, $2 \mathrm{H}), 2.16(\mathrm{~d}, J=1.3 \mathrm{~Hz}, 3 \mathrm{H}), 2.09-1.97(\mathrm{~m}, 1 \mathrm{H}), 1.85$ (ddd, $J=13.6,3.9,2.9 \mathrm{~Hz}, 1 \mathrm{H}$ ), 1.77 (ddd, $J=12.0,7.2$, $5.1 \mathrm{~Hz}, 1 \mathrm{H}), 1.58-1.51(\mathrm{~m}, 1 \mathrm{H}), 1.41-1.36(\mathrm{~m}, 1 \mathrm{H}), 1.36$ $(\mathrm{s}, 3 \mathrm{H}), 1.14(\mathrm{~s}, 3 \mathrm{H}), 1.12(\mathrm{~d}, J=6.8 \mathrm{~Hz}, 3 \mathrm{H}), 1.07(\mathrm{t}, J=$ $7.6 \mathrm{~Hz}, 3 \mathrm{H}), 0.91(\mathrm{~s}, 3 \mathrm{H}), 0.85(\mathrm{~d}, J=6.9 \mathrm{~Hz}, 3 \mathrm{H}) ;{ }^{13} \mathrm{C}$ NMR $\left(100 \mathrm{MHz}, \mathrm{CDCl}_{3}\right) \delta 211.3,166.6,162.9,140.1$, $126.9,113.9,97.2,88.9,87.7,78.3,74.2,73.2,49.6,38.8$, $37.4,33.9,29.1,28.9,28.14,28.10,25.5,25.1,19.0,17.0$, 16.0, 11.9; IR (neat) v 3368, 2956, 2924, 2854, 1717, 1649, $1460,1379,1260,1215,1142,1098,1073,1024,980,852$, 795, 701; HRMS (ESI) found $487.2693[\mathrm{M}+\mathrm{Na}]^{+}$, calcd 487.2672 for $\mathrm{C}_{26} \mathrm{H}_{40} \mathrm{O}_{7} \mathrm{Na}^{+}$.

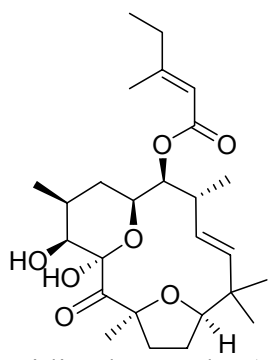

Data are (20c oxidized product): tlc $R_{f}=0.58$ (hexanes/EtOAc, 2.5:1); $[\alpha]^{20}{ }_{\mathrm{D}}=-16.0^{\circ},[\alpha]^{20}{ }_{365 \mathrm{~nm}}=-$ $185.6^{\circ}$ (c 0.063, $\left.\mathrm{CHCl}_{3}\right) ;{ }^{1} \mathrm{H}$ NMR $\left(400 \mathrm{MHz}, \mathrm{CDCl}_{3}\right) \delta$ $6.25(\mathrm{~s}, 1 \mathrm{H}), 5.77(\mathrm{br} \mathrm{s}, 1 \mathrm{H}), 5.48(\mathrm{~d}, J=15.2 \mathrm{~Hz}, 1 \mathrm{H})$, $5.40(\mathrm{dd}, J=15.2,9.1 \mathrm{~Hz}, 1 \mathrm{H}), 4.93(\mathrm{dd}, J=7.8,2.8 \mathrm{~Hz}, 1$ H), 4.32 (dt, $J=12.3,2.8 \mathrm{~Hz}, 1 \mathrm{H}), 3.96(\mathrm{t}, J=7.2 \mathrm{~Hz}, 1$ H), 3.86 (br s, $1 \mathrm{H}), 3.53$ (br s, $1 \mathrm{H}), 2.54-2.46(\mathrm{~m}, 1 \mathrm{H})$, $2.34(\mathrm{dq}, J=12.5,8.1 \mathrm{~Hz}, 1 \mathrm{H}), 2.22-2.16(\mathrm{~m}, 1 \mathrm{H}), 2.17$ (q, $J=7.4 \mathrm{~Hz}, 1 \mathrm{H}), 2.14$ (br s, $3 \mathrm{H}), 2.03-1.92(\mathrm{~m}, 2 \mathrm{H})$, 1.81 (ddd, $J=12.5,7.5,5.7 \mathrm{~Hz}, 1 \mathrm{H}), 1.63(\mathrm{q}, J=12.3 \mathrm{~Hz}$, $1 \mathrm{H}), 1.42(\mathrm{~s}, 3 \mathrm{H}), 1.18(\mathrm{~s}, 3 \mathrm{H}), 1.14-1.08(\mathrm{~m}, 1 \mathrm{H}), 1.08$ $(\mathrm{t}, J=7.4 \mathrm{~Hz}, 3 \mathrm{H}), 1.00(\mathrm{~d}, J=6.9 \mathrm{~Hz}, 3 \mathrm{H}), 0.97(\mathrm{~d}, J=$ 
$6.9 \mathrm{~Hz}, 3 \mathrm{H}), 0.94(\mathrm{~s}, 3 \mathrm{H}) ;{ }^{13} \mathrm{C} \mathrm{NMR}\left(125 \mathrm{MHz}, \mathrm{CDCl}_{3}\right) \delta$ 208.7, 167.1, 162.2, 135.1, 130.0, 114.4, 97.0, 91.1, 89.2, $76.9,70.9,68.8,41.7,38.7,36.1,33.8,29.2,28.5,26.0$, $25.2,24.42,24.39,20.4,18.9,17.3,11.9$; IR (neat) $\vee 3360$, 2962, 2926, 2872, 1714, 1650, 1457, 1377, 1311, 1263, 1216, 1143, 1095, 1030, 995, 977, 866, 803; HRMS (ESI) found $487.2683[\mathrm{M}+\mathrm{Na}]^{+}$, calcd 487.2672 for $\mathrm{C}_{26} \mathrm{H}_{40} \mathrm{O}_{7} \mathrm{Na}^{+}$. 